\title{
VOTING BY SIMULTANEOUS VETOES *
}

\author{
Margarita Kirneva ${ }^{\dagger}$ And Matías NúÑEZ $Z^{\ddagger}$
}

MAY 2021

\begin{abstract}
We propose the first class of simultaneous voting mechanisms in which each Nash equilibrium is coalition-proof. These mechanisms hence prevent the coordination failures which arise when some (coalition of) voters could have induced an outcome that they all prefer to the equilibrium outcome had they agreed on a common strategy. In each of these mechanisms, some voter(s) has the right to veto a list of alternatives. For each specification of the veto rights, each of these mechanisms implements a Veto by random priority rule introduced by Moulin [1981]. We then discuss necessary conditions for arbitrary mechanisms to implement a Pareto efficient rule ensuring that each equilibrium is coalition-proof. We show that the presence of veto rights in the mechanism is unavoidable to achieve this demanding implementation notion.
\end{abstract}

JEL Codes: D71, D72.

Keywords: Implementation, Voting, Vetoes, Coalition Formation, Efficiency.

\section{Introduction}

Selection committees are involved in many key decisions both in academia and in the public and the private sectors. In the former, appointments tend to be made by committees whereas in the latter boards of directors tend to manage corporate governance. Interest rates are also

\footnotetext{
${ }^{\star}$ We would like to thank Kfir Eliaz, Yukio Koriyama, Jean-François Laslier, Hervé Moulin, Pietro Ortoleva, Ludovic Renou and Alessandro Riboni as well as seminar participants at the CREST Political economy working group and at Economic Theory seminar at Paris 1. This paper was circulated under the title "On Coordination in Selection Committees". Financial support from the project ANR-11-IDEX-0003/Labex Ecodec/ANR-11-LABX0047 is gratefully acknowledged.

${ }^{\dagger}$ CREST, CNRS, Ecole polytechnique, GENES, ENSAE Paris, Institut Polytechnique de Paris, 91120 Palaiseau, France.

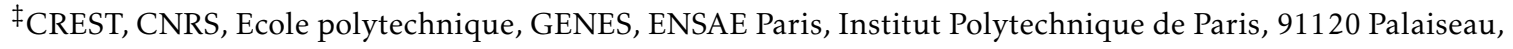
France.
} 
often determined through committees such as the LIBOR and EURIBOR benchmark rates. Likewise, the European Central Bank and the FED decide on monetary policies through committees. ${ }^{1}$ Behind this extended use of committees often lies the idea that taking into account the opinion of several voters can improve the quality of the final decisions. Despite the ubiquity of committees, it is commonly agreed among economists that selection committees might generate inefficiencies. There are several reasons behind these inefficiencies ${ }^{2}$ such as poor information revelation, delays or status-quo bias. Moreover, classical results support this suspicion of inefficiency since no usual voting rule is strategy-proof or ensures that all equilibria are well behaved (see the review of the literature). In particular, no usual rule (theoretically) guarantees that every equilibrium outcome is Pareto-efficient unless some restrictions are imposed. ${ }^{3}$ Moreover, coordination failures among voters might lead to the election of a minority preferred candidate, due to poor incentives for coordination.

A usual manner to achieve this goal is to introduce a domain restriction, such as singlepeakedness, that allows to design attractive rules and mechanisms such as the median rules (see Moulin [1980] for a classic treatment and Núñez and Xefteris [2017] for Nash implementation in this setting). While this restriction is reasonable and can be induced in lab experiments, in real-life settings, one cannot control the preferences of the voters. Thus, it seems important to design efficient voting mechanisms that do not rely in this constraint. We follow this approach (that is, imposing no restriction over the voters' preferences) and focus on the design of voting mechanisms that ensure that every equilibrium is coalition-proof. ${ }^{4}$ This means that, in equilibrium, no coalition of voters can profitably deviate by communicating to devise a common strategy. In the mechanisms we propose, the simultaneous veto (SV) mechanisms, the strategy of each voter is simply to veto a list of alternatives, after which the winning alternative is randomly selected from the nonvetoed alternatives. Assuming that voters' preferences over lotteries respect the condition of stochastic dominance,

\footnotetext{
${ }^{1}$ See Riboni and Ruge-Murcia [2010] and Riboni and Ruge-Murcia [2014] for a strategic analysis of voting in monetary policy committees.

${ }^{2}$ See Caillaud and Tirole [2007] and Visser and Swank [2007] for the role of information sharing in committees and Bayesian persuasion.

${ }^{3}$ In the extreme case of two voters, no Pareto efficient rule is Nash implementable. With three or more voters, some rules of interest are implementable (see Hurwicz and Schmeidler [1978] and Maskin [1999]) but none of them among the usual voting rules. A noteworthy exception is the kingmaker mechanism which implements Pareto-efficient alternatives, as we discuss in the main text.

${ }^{4}$ Our results rely on complete information since we find it to be an appropriate assumption for small committees in which members have accurate beliefs over the preferences of the rest of the voters (see De Clippel et al. [2014] for a similar argument in the selection of arbitrators)
} 
these mechanisms ensure that every equilibrium is Pareto-efficient. ${ }^{5}$ Hence, our work is in the line of Bouton et al. [2018], who argue in favor of majority voting with veto power for two alternatives and of Laslier et al. [2021], who show how simultaneous vetoes can lead to efficient agreements in two player settings. It is also related to Bogomolnaia et al. [2021] that focuses on the worst case welfare a voter can secure against unanimously adversarial others in voting and bargaining environments. ${ }^{6}$

The first part of this work is concerned with the equilibrium properties of SV mechanisms. ${ }^{7}$ We show that at each equilibrium no two voters veto the same alternative, thus any equilibrium is always deterministic. This, is turn, implies each voter is pivotal in equilibrium and hence only Pareto-efficient alternatives can be elected. ${ }^{8}$ We then characterize the set of equilibrium outcomes of $S V$ mechanisms. Denoting by $v_{i}$ the number of alternatives that voter $i$ can veto, it is intuitive to see that the outcome can never be among the $v_{i}$ worst alternatives for voter $i$, thus giving each voter a minimal level of satisfaction. However, we show that in equilibrium voters coordinate their vetoes to prevent alternatives which are bad enough for the different coalitions. Our main result can thus be stated as follows: an SV mechanism with veto vector $v$ Nash implements the Veto by random priority rule (or VRP) with veto vector $v$. For any veto vector, any alternative in the VRP rule can be justified (see Moulin [1983]) through the following naive algorithm: the voters assign their vetoes sincerely and sequentially one-at-a-time. ${ }^{9}$ A key property of our result is that each Nash equilibrium is also coalition-proof (and by definition each coalition-proof equilibrium is Nash). We refer to this double implementation (i.e., in Nash and coalition-proof) as coalitional implementation. ${ }^{10}$

\footnotetext{
${ }^{5}$ The observation that vetoes can lead to Pareto-efficient outcomes is present in the literature dealing the unanimity rule and two alternatives; however, the main novelty of our work is the use of vetoes in settings with many alternatives combined with lotteries as off-equilibrium threats. Our mechanisms select a single alternative in equilibrium with off-equilibrium lotteries. See Brandt [2015] for examples of such rules that satisfy a version of strategy-proofness and Brandt et al. [2016] for a discussion.

${ }^{6}$ See also Macé and Treibich [2021] who studies cooperation among heterogeneous voters and argues that veto power might be needed to induce efficiency.

${ }^{7}$ We work under the assumption that the sum of the veto rights is equal to the number of alternatives minus one to ensure that there is always some nonvetoed alternative and that the equilibrium outcome is deterministic.

${ }^{8}$ As we discuss in Section 4, few mechanisms are known to implement a Pareto efficient SCR except dictatorships, modulo/integer games and the kingmaker games. More none of the classical voting rules such as Borda or Plurality satisfy the stringent condition of Maskin monotonicity (see Jackson [2001] for a discussion).

${ }^{9}$ This rule is referred in the literature as the veto core correspondence. The set of alternatives that the VRP rule selects corresponds to the different orderings of the vetoes, which justifies the appeal of this rule.

${ }^{10}$ In a coalition-proof equilibrium, no coalition can profitably deviate. Thus, coalition-proofness and communication are deeply linked: voters' behavior occurs as if pre-play communication takes place where the voters can make binding commitments on the ballots they will choose. See Bernheim et al. [1987] for the initial work on this equilibrium notion. Observe that the equilibrium in which all voters vote for the same alternative under the
} 
The second part of the paper focuses on coalitional implementation through arbitrary mechanisms. We provide the necessary conditions that the veto structure ${ }^{11}$ of the implementing mechanism must satisfy in order to implement a Pareto efficient rule. As we show, the sum of the veto rights of any partition of the voters adds up to $k-1$, where $k$ is the number of alternatives. This means that the veto rights are key to ensure this implementation notion.

We then explore the rules that can be coalitionally implemented. Note that any dictatorship (where one voter has all veto rights) satisfies these properties. However, the class of rules satisfying these conditions includes several rules in which all voters have (almost-) identical veto rights.

We consider implementation through mechanisms that exhibit No Gains from Cooperation (NGC): in these mechanisms, a coalition has no more veto power than the union of its members, as in the SV mechanisms. This condition can be thought as an idea of simplicity of the mechanism: in any neutral mechanism satisfying it, the veto powers of the voters suffice to describe the veto power of any coalition in the game. Under this condition, we are able to show that if a rule $f$ is coalitionally implementable then $f \supseteq$ VRP for some vector $v$. A similar result applies when there are no less alternatives than voters: at least some voter has veto rights in the implementing mechanism.

If there are less alternatives than voters, one can achieve coalitional implementation without allocating veto rights to any voter but rather delegate the right to veto to coalitions. This is the case in the partial vetoes mechanism that we build to implement the majority rule in a two alternative setting. In this mechanism, the coalitions are endogenously formed in order to obtain the veto rights; it is hence related to the coalition formation literature. ${ }^{12}$ This is, to the best of our knowledge, the first voting mechanism that implements the majority rule. Indeed, recent work by Xiong [2021] has shown that no simple mechanism can implement this rule. We also show how to extend this mechanism to any number of alternatives.

In the final set of results, we provide an impossibility result that shows that coalitional implementation is not possible via deterministic mechanisms when there are more alterna-

plurality rule (previously described) need not be coalition-proof since some coalition of voters might prefer to deviate. In general, the plurality rule fails to admit coalition-proof equilibria (see Messner and Polborn [2007]).

${ }^{11}$ More precisely, as argued by Moulin [1981] and Moulin [1982], any rule assigns some veto power to each voter and to each coalition - the right to prevent any set of alternatives of a given size (possibly zero) from being elected. We call the collection of such veto powers the veto structure of a rule.

${ }^{12}$ Coalition formation studies coalitions as equilibria of non-cooperative games. See Nash et al. [2012] for recent work on coalition formation and Ray and Vohra [2015] for an excellent account. 
tives than voters and via deterministic NGC mechanisms in general settings; hence, using off-equilibrium lotteries is needed to ensure coordination.

Section 2 presents a review of the literature while Section 3 introduces the setting and the definition of coalitional implementation. Section 4 shows that simultaneous veto mechanisms Nash implement the VRP rules. Finally, Section 5 considers coalitional implementation through arbitrary mechanisms.

\section{Review of the literature}

Our paper is at the intersection of the literature on committee design and the literature on implementation.

Regarding committee design, the classic literature focuses on strategy-proofness while the recent one is more concerned with equilibrium predictions. Moulin [1980] focuses on both the median rule and the strategy-proof rules in the single-peaked domain. Border and Jordan [1983] and Barberà et al. [1993] design strategy-proof rules on multidimensional settings. More closely related to our work, Gershkov et al. [2017] derive rules that maximize the collective welfare in equilibrium whereas Núnez and Xefteris [2017] design approval mechanisms that Nash implement the median rule. The assumption of single-peakedness is meaningful in many settings of interest such as the provision of public goods. Moreover, this condition is technically important since the notion of the majority preference is simply undefined without restrictions over preferences (see Cox and Shepsle [2007]). However, several settings of interest are multidimensional per se, including the location of headquarters (quality of labor supply vs. taxation conditions), recruitments in an academic department (quality vs. fitness of the candidate), and project investments (short-term vs. long term profitability) among others. In this work, we implement rules, the VRP rules, that can be of use in these multidimensional settings. ${ }^{13}$ However, in these settings, it seems difficult to rely on the dimensions of the space to design the voting procedure since it may be difficult to specify the dimensions that matter at least ex-ante. ${ }^{14}$

Regarding implementation, our paper is related to the classic and to the most recent literature on vetoes and implementation. After the pioneering works by Hurwicz and Schmeidler [1978] and Maskin [1999] that propose the idea of implementation and provide the

\footnotetext{
${ }^{13}$ See Gershkov et al. [2019] for a recent contribution in voting in a multidimensional setting.

${ }^{14}$ Related to our work are the contributions of Bierbrauer and Hellwig [2016] which shows that coalition-proof incentive mechanisms for public good provision are voting mechanisms.
} 
first results dealing with monotonicity, Dutta and Sen [1991] and Moore and Repullo [1990] give necessary and sufficient conditions for implementation with two and more players. ${ }^{15}$ More recently, in two-voter settings, De Clippel et al. [2014] argues that dynamic vetoes can be of use for improving coordination whereas Laslier et al. [2021] prove that simultaneous veto mechanisms lead to the implementation of Pareto-and-veto rules. Bouton et al. [2018] argues that in status-quo elections, majority rules with veto are superior to the usual unanimity rule. Bogomolnaia et al. [2021] analyze bargaining settings from the perspective of vetoes. Our VRP rules coincide with Pareto-and-veto rules in settings with two voters and coincide with the "veto core" correspondence in Moulin [1983]. Mueller [1978] proposes a dynamic veto mechanism for public good division, and shows that this mechanism leads only to Pareto-dominant outcomes. Finally, our work is related to the works on implementation in which lotteries are used as off-equilibrium threats. Bochet [2007] and Benoît and Ok [2008] show that a rule is Maskin monotonic if and only if it Nash implementable when lotteries are introduced off-equilibrium. Their works are mainly concerned with integer games and the idea that the usual condition of no-veto power (needed in the classic characterization by Maskin [1999]) can be dispensed. Our work is concerned with the design of voting mechanisms that ensure coordination that rely on off-equilibrium threats. Several works have tried to combine Nash-implementation with other notions of equilibria (as we do). Maskin [1978] and Suh [1997] consider implementation under Nash and strong Nash equilibrium; their results are negative in the sense that few interesting rules are implementable according to their definition. Likewise, since many strategy-proof mechanisms have multiple Nash equilibria, Saijo et al. [2007] propose the notion of secure implementation, that is Nashimplementation and implementation in dominant strategies. Our idea is similar: we focus on double implementation in Nash and coalition-proof equilibrium.

Several papers characterize voting rules by their associated veto functions and give the conditions that the veto function of a rule should satisfy to achieve some desirable properties. Moulin [1981] and Moulin [1982] introduce the proportional veto principle and show that such a rule is both stable and leads to the smallest outcome set among stable anonymous rules (see Ianovski and Kondratev [2020] for a computation of the size of the proportional veto core). Moreover a veto function that is close to proportional is required for any rule to be partially implementable in Strong Nash. The necessary conditions obtained in Theorem 2 have some similarities with the conditions given by Theorem 1 in Moulin [1982].

\footnotetext{
${ }^{15}$ See also Dutta and Sen [2012] for implementation with partially honest players.
} 
Keiding and Peleg [2002] provide the necessary conditions for a social choice rule to be implementable in coalition-proof equilibria in terms of effectivity functions, the reformulation of which to veto functions is straightforward. By considering double implementation in both Nash and coalition-proof equilibria we restrict the set of possible rules that can be implemented in a way that avoids bad equilibria.

\section{Setting for Committee Design}

A committee $I=\{1, \ldots, n\}$ of voters with $n \geq 3$ needs to choose an alternative out of a finite set $A$ of alternatives. While many results require that $|A|=k>n$, we are also concerned with situations with $k \leq n .{ }^{16}$ Each voter $i \in I$ has a strict, binary and transitive preference relation $>_{i}$ defined over $A$. For each $i$, let $R_{i}$ be the class of possible preference relations for voter $i$. Let $>=\left(>_{1}, \ldots,>_{n}\right) \in R \equiv \prod_{i \in N} R_{i}$ be a preference profile. For each $i \in I,>_{i} \in R_{i}$ and $a \in A$, let $L\left(a,>_{i}\right) \equiv\left\{b \in A \mid a>_{i} b\right\}$ be the lower contour set of $a$ at $>_{i}$ with cardinal $l\left(a,>_{i}\right)$. We denote by $C^{I}$ the set of possible coalitions in $I$. For any coalition $J \in C^{I}$ we denote by $R_{J} \equiv \prod_{i \in J} R_{i}$ the set of possible joint preference profiles of the voters in the coalition $J$. For $J \in C^{I},>_{J} \in R_{J}$ and $a \in A$, let $L\left(a,>_{J}\right) \equiv\left\{b \in A \mid b \in L\left(a,>_{i}\right)\right.$ for some $\left.i \in J\right\}$ be the lower contour set for coalition $J$ of $a$ for $>_{J}$ with cardinal $l\left(a,>_{J}\right)$. Note that for each $J \in C^{I}$ and each $a \in A$, $L\left(a,>_{J}\right)=\bigcup_{i \in J} L\left(a,>_{j}\right)$.

A social choice rule (SCR) is a function $f: R \rightrightarrows A$ that associates a set $f(>)$ of alternatives to every $>\in R$. A SCR $f$ satisfies Maskin monotonicity iff for all $>,>^{\prime} \in R$ and $x \in A$ with $L\left(x,>_{i}\right) \subseteq L\left(x,>_{i}^{\prime}\right)$ for all $i \in I$, we have $x \in f(>) \Rightarrow x \in f\left(>^{\prime}\right)$. An alternative $x$ is Paretoefficient in the preference profile $>$ if there is no $y \in A$ such that $y>_{i} x$ for each $i \in N$. A SCR $f$ is Pareto efficient if $f(>)$ only contains Pareto efficient alternatives for any $>\in R$.

\subsection{Mechanisms}

We let $\Delta$ denote the set of lotteries over $A$ with $\Delta=\left\{\beta: A \rightarrow[0,1] \mid \sum_{a \in A} \beta(a)=1\right\}$. For each $\beta \in \Delta, \operatorname{supp}(\beta)$ denotes the support of $\beta$, that is the alternatives that are selected with positive probability according to $\beta$. For each set $X \subseteq A$, we write unif $(X)$ to denote the uniform lottery over $X$, that is $\operatorname{unif}(X)=\left\{\beta \in \Delta \mid \beta(x)=\frac{1}{|X|}\right.$ for any $\left.x \in X\right\}$. A mechanism is a function $g: M \rightarrow \Delta$ that assigns to every $m \in M$ a unique element of $\Delta$, where $M=\prod_{i \in I} M_{i}$, and $M_{i}$ is the strategy space of voter $i$. The list $m \in M$ is written as $\left(m_{i}, m_{-i}\right)$, where $m_{-i}=$

\footnotetext{
${ }^{16}$ See Laslier et al. [2021] for a related analysis with two voters.
} 
$\left(m_{1}, \ldots, m_{i-1}, m_{i+1}, \ldots, m_{n}\right) \in M_{-i}=\prod_{j \neq i} M_{j}$. Let $g(M)$ be the range of the mechanism $g$ with $g(M)=\{\beta \in \Delta \mid \exists m \in M$ s.t. $g(m)=\beta\}$. We only consider mechanisms with a finite range. Let $g\left(M_{i}, m_{-i}\right)=\left\{\beta \in \Delta \mid g\left(m_{i}, m_{-i}\right)=\beta\right.$ for some $\left.m_{i} \in M_{i}\right\}$ be the attainable set of voter $i$ at $m_{-i}$, in other words, the set of lotteries that voter $i$ can induce when the other voters play $m_{-i}$.

Given a mechanism $g$ and a coalition $J$, we let $M_{J}$ denote its strategy space with $M_{J}=$ $\prod_{i \in J} M_{i},\left(m_{J}, m_{-J}\right)$ stand for the message profile where coalition $J$ plays strategy $m_{J}$ and coalition $I \backslash J$ plays strategy $m_{-J}$ and $\left(m_{J}^{\prime}, m_{-J}\right)$ stand for the message profile obtained from $\left(m_{J}, m_{-J}\right)$ by replacing the strategy $m_{J}$ by the strategy $m_{J}^{\prime}$. The attainable set of coalition $J$ at $m_{-J}$ is defined as $g\left(M_{J}, m_{-J}\right)$, in a similar manner to the attainable set of a voter.

\subsection{Preferences over lotteries}

We assume that preferences over lotteries are derived from the preferences over alternatives through preference extensions. We say that $\geq_{i}^{*}$ is an extension of $>_{i}$ if, for any pair $x, y \in A$, $x>_{i} y \Longrightarrow x>_{i}^{*} y$. For each mechanism $g: M \rightarrow \Delta$, we write $R_{g(M)}$ the class of preferences over the lotteries in $g(M)$. An element of $R_{g(M)}$ is denoted by $\geq_{i}^{*}$ and $\geq^{*}$ be a profile of preference extensions. Let $\kappa\left(>_{i}\right) \in R_{g(M)}$ be a set of admissible preference extensions of voter $i$ associated to $>_{i}$. Similarly, $\kappa(>)$ is the set of admissible preference preferences associated to $>$.

We assume that preferences over lotteries satisfy stochastic dominance (SD). We follow the definition of Bochet and Maniquet [2010]: for any $i \in N$ and any $j=0, \ldots, k$, let $p_{j}: R_{i} \rightarrow A$ be defined by:

$$
p_{j}\left(>_{i}\right)=a \Longrightarrow l\left(a,>_{i}\right)=k-j
$$

that is, $p_{1}\left(>_{1}\right)$ is the preferred alternative of $>_{i}, p_{2}\left(>_{1}\right)$ is her second preferred and so on. Then, for all $\geq^{*} \in \mathcal{K}(>)$, if two lotteries $\beta$ and $\gamma$ are such that for each $j^{*} \in\{1, \ldots, k\}$, $\sum_{j \leq j^{*}} \beta\left(p_{j}\left(>_{i}\right)\right) \geq \sum_{j \leq j^{*}} \gamma\left(p_{j}\left(>_{i}\right)\right)$ then $\beta \geq_{i}^{*} \gamma$ and $\beta>_{i}^{*} \gamma$ if one inequality is strict.

The second condition, Priority Extension, deals with the richness of the domain of preference extensions. For any lottery $\beta \in \Delta$ and each $x$ with $p_{j^{*}}\left(>_{i}\right)=x$, we write $\beta^{+}[x]=$ $\sum_{j<j^{*}} \beta\left(p_{j}\left(>_{i}\right)\right)$ to refer to the probability, according to $\beta$, of obtaining an alternative preferred to $x$ according to $>_{i}$.

Let $\geq_{i}^{*}$ be an extension of $>_{i}$ and $x \in A, \geq_{i}^{*}$ is a priority extension (PREX) for $x$ iff given two lotteries $p, q \in \Delta$, if $p^{+}[x]>0$ and $q^{+}[x]=0$, then $p>_{i}^{*} q$. We say that a domain $\kappa$ satisfies PREX in $\Delta$ iff for all $>_{i} \in R_{i}$ and for each $x \in A$, there is some $\geq_{i}^{*} \in \kappa\left(>_{i}\right)$ that satisfies PREX for $x$ (see Laslier et al. [2021] for a discussion on this condition). 


\subsection{Nash Implementation}

Given a mechanism $g: M \rightarrow \Delta$, the strategy profile $m \in M$ is a Nash equilibrium of $g$ at $\geq^{*}$ if there is no $i \in I$ with $g\left(m_{i}^{\prime}, m_{-i}\right)>_{i}^{*} g(m)$ for some $m_{i}^{\prime} \in M_{i}$. The set $B R\left(\geq_{i}^{*}, m_{-i}\right)$ denotes the best responses of a voter with type $\geq_{i}^{*}$ under the mechanism $g$ when her opponents play according to $m_{-i}$. Let $N^{g}\left(\geq^{*}\right)$ be the set of Nash equilibria of $g$ at $\geq^{*}$. In the sequel, we consider mechanisms that are deterministic or deterministic-in-equilibrium (DE). A mechanism $g$ is deterministic if for any $m \in M, g(m) \in A$. A mechanism $g$ is DE in the domain $\kappa(>)$ if for any $>\in R$ and any $\geq^{*} \in \kappa(>)$ and any $m \in N^{g}\left(\geq^{*}\right), g(m) \in A$.

The mechanism $g$ implements the SCR $f$ in Nash equilibria in the domain $\kappa(>)$ if for each $>\in R$ and each $\geq^{*} \in \mathcal{K}(>)$, (i) for each $x \in f(>)$, there exists $m \in N^{g}\left(\geq^{*}\right)$ such that $g(m)=\{x\}$ and (ii) for any $m \in N^{g}\left(\geq^{*}\right), g(m)=\{y\}$ for some $y \in f(>)$. The SCR $f$ is Nash implementable if there exists a mechanism that implements $f$ in Nash equilibria.

\subsection{Coalition-proof and Coalitional Implementation}

The strategy $m_{J}^{\prime}$ is a self-enforcing deviation for coalition $J$ at profile $m$ if $g\left(m_{J}^{\prime}, m_{-J}\right)>_{j}^{*} g(m)$ for all $j \in J$ and there is no $H \in C^{J}$ with some $m_{H}^{\prime \prime}$ such that $g\left(m_{H}^{\prime \prime}, m_{J \backslash H}^{\prime}, m_{-J}\right)>_{h}^{*} g\left(m_{J}^{\prime}, m_{-J}\right)$ for all $h \in H$ for some preference extension $\geq^{*} \in \kappa(>)$. The strategy profile $m \in M$ is a coalitionproof equilibrium of $g$ at $\geq^{*}$ in the domain $\kappa(>)$ if no coalition $J \in C^{I}$ has a self-enforcing deviation. ${ }^{17}$ Observe that since any unilateral deviation by a single voter is self-enforcing, any coalition-proof equilibrium is a Nash equilibrium.

Let $C^{g}\left(\geq^{*}\right)$ be the set of coalition-proof equilibria of $g$ at $\geq^{*}$. By definition $C^{g}\left(\geq^{*}\right) \subseteq$ $N^{g}\left(\geq^{*}\right)$. The mechanism $g$ implements the SCR $f$ in coalition-proof equilibria in the domain $\kappa(>)$ if for each $>\in R$ and $\geq^{*} \in \kappa(>)$, (i) for each $x \in f(>)$, there exists $m \in C^{g}\left(\geq^{*}\right)$ such that $g(m)=\{x\}$ and $(i i)$ for any $m \in C^{g}\left(\geq^{*}\right), g(m)=\{y\}$ for some $y \in f(>)$. The SCR $f$ is coalitionproof Nash implementable if there exists a mechanism that implements $f$ in coalition-proof equilibria.

A SCR $f$ is coalitionally implementable at the domain $\kappa(>)$ if $f$ is implementable in both Nash and coalition-proof equilibria by some mechanism $g$ at the domain $\kappa(>)$ such that: for

\footnotetext{
${ }^{17}$ This definition and the classical definition for coalition-proof Nash are equivalent. For self-enforcing deviation of coalition we need to consider all the coalitions of smaller size, so we can write it in the recursive form. Consider this step from the original definition: Then for a game $\left\langle I, M, P^{*}\right\rangle$ with $|I|=n, m \in M$ is self-enforcing if for all $J \subset I, m_{J}$ is a coalition-proof equilibrium in the game $\left\langle J, M_{J}, P_{J}^{*}\right\rangle$. This is exactly the same as to say that there is no coalitional self-enforcing deviations. Also we have included already in the definition of coalitional self-enforcing deviation that there should be no other such deviation which is better for all the voters of coalition which gives the second part from the classical definition of coalition-proof Nash.
} 
each $>\in R$ and for each $\geq^{*} \in \kappa(>)$ (1) $N^{g}\left(\geq^{*}\right) \neq \emptyset$ and $C^{g}\left(\geq^{*}\right) \neq \emptyset$, (2) $N^{g}\left(\geq^{*}\right)=C^{g}\left(\geq^{*}\right)$, and (3) $f(>)=\bigcup_{m \in N^{g}\left(\geq^{*}\right)} g(m)$.

\section{Coalitional implementation via simultaneous vetoes}

This section presents the simultaneous veto mechanisms and introduces the first main result of this work: simultaneous veto mechanisms Nash implement Veto by random priority rules. After defining the Veto by random priority rule, it introduces the simultaneous veto mechanisms, discusses its basic properties and shows the implementation argument. The final part shows that the simultaneous veto mechanisms coalitionally implement the VRP rules.

\subsection{Veto by random priority rule}

Let $v=\left(v_{i}\right)_{i \in N}$ be a set of non-negative integers such that

$$
\sum_{i \in N} v_{i}=k-1
$$

From now on, we refer to any vector $v$ that satisfies (1) as the vector of veto rights (or simply veto vector) and to $v_{i}$ as the veto rights of voter $i$. Similarly, $v_{J}=\sum_{i \in J} v_{i}$ denotes the veto rights of coalition $J$.

For each profile $>\in R$, the veto by random priority rule with veto vector $v$, denoted $\mathrm{VRP}_{v}$, selects all the alternatives with a lower contour set greater than $v_{J}$ for each coalition $J$, that is:

$$
\{a\} \in \operatorname{VRP}_{v}(>) \Longleftrightarrow \text { for each coalition } J \in C^{I}, l\left(a,>_{J}\right) \geq v_{J}
$$

When a voter has veto power $v_{i}$, the VRP rule never selects an alternative among her worst $v_{i}$ alternatives. Similarly, when a coalition has veto power $v_{J}$ the outcome is never among the worst $v_{J}$ alternatives of the coalition. By doing so, the VRP rule ensures a minimal level of satisfaction to each coalition of voters.

This rule is discussed in depth by Moulin [1983] and it is referred as the Veto core correspondence. Note that with 2 voters, this rule coincides with the Pareto-and-veto rules (see Laslier et al. [2021]). In a setting with three voters, the VRP rule can be illustrated as follows. 
Letting $I=\{1,2,3\}$, the set $C^{I}$ of possible coalitions equals:

$$
C^{I}=\{\{1\},\{2\},\{3\},\{1,2\},\{1,3\},\{2,3\},\{1,2,3\}\}
$$

The definition of $\mathrm{VRP}_{v}$ entails here that for any veto vector $v$, an alternative $a$ is selected if and only if it satisfies the following inequalities:

$$
\begin{aligned}
& \underbrace{l\left(a,>_{i}\right) \geq v_{i} \text { for each } i \in I}_{\text {individual veto power }}, \quad \underbrace{l(a,>) \geq v_{1}+v_{2}+v_{3}}_{\text {Pareto efficiency }} \\
& \underbrace{\text { and } l\left(a,>_{J}\right) \geq v_{J} \text { for each } J \in\{\{1,2\},\{1,3\},\{2,3\}\}}_{\text {veto coordination }} .
\end{aligned}
$$

The two first sets of inequalities convey a clear message: any alternative selected by the $\mathrm{VRP}_{v}$ rule is Pareto-efficient and, for each voter $i$, the alternative does not belong to her worst $v_{i}$ alternatives. The third set of inequalities ensures that communication among voters is effective and no veto is wasted. Indeed, $l\left(a,>_{J}\right) \geq v_{J}$ implies that there are at least $v_{J}$ alternatives less preferred than $a$ for the different members of the coalition $J$.

The definition of the $\mathrm{VRP}_{v}$ rule, stated by (2), relies on the cardinal of the different lower contour-sets. While being compact such formulation is not the most intuitive. We now provide an alternative formulation of this rule which gives additional intuition for how the alternatives are selected. To state this formulation, we introduce the following notation: for each veto vector $v$ satisfying (1), we denote by $\Pi(I, v)$ the set of all possible orderings of the voters' vetoes, where each voter appears exactly $v_{i}$ times. The generic element of $\Pi(I, v)$ is denoted by $\pi$. We refer to $\pi_{j}$ as the $j^{\prime}$ th element in $\pi$. For example, if $I=\{1,2,3\}$ and the veto vector equals $v=(3,2,1)$ with 7 alternatives, then one possible ordering can be $(1,2,1,1,3,2)$ so that voter 1 appears in positions 1, 3 and 4, voter 2 in position 2 and 6 and voter 3 in the position 5 of $\pi$.

For each veto vector $v$ satisfying (1) and each ordering $\pi \in \Pi(I, v)$, let $\operatorname{VoSP}_{\pi, v}$ denote the Veto by one-at-a-time successive priority rule, which is determined through the following algorithm: 


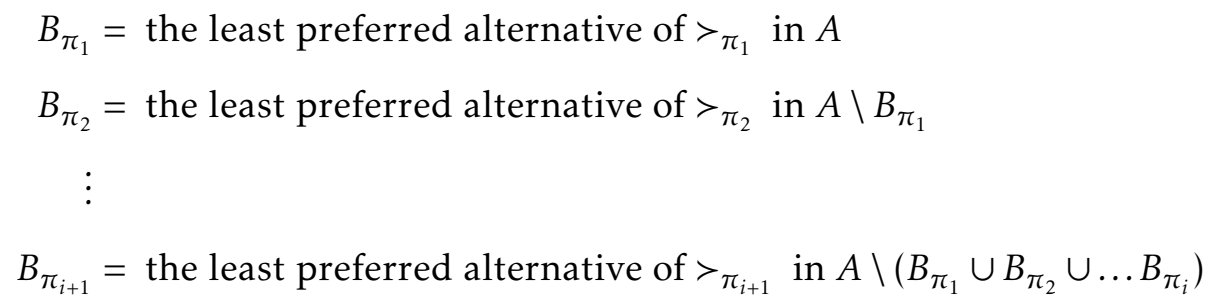

The previous algorithm singles out an alternative for each specification of the veto rights: the set $A \backslash\left(B_{\pi_{1}} \cup B_{\pi_{2}} \cup \ldots B_{\pi_{k-1}}\right)$ is a singleton. We denote this alternative as $\operatorname{VoSP}_{\pi, v}(>)$. This alternative is well-defined for each $>\in R$ and is Pareto-efficient (see Moulin [1983]).

Proposition 1. (Moulin [1983], Theorem 4) For each veto vector $v$ and each preference profile $>\in R$,

$$
V R P_{v}(>)=\bigcup_{\pi \in \Pi(I, v)} \operatorname{VoS} P_{\pi, v}(>) .
$$

The former proposition claims that the set of alternatives selected by the veto by random priority rule, $\mathrm{VRP}_{v}$, coincides with the set of alternatives the one can get applying $\operatorname{VoSP}_{\pi, v}$ to any possible ordering of the vetoes.

Remark 1: For each preference profile $>\in R$ and each veto vector $v$, the rule $\operatorname{VRP}_{v}(>)$ is well-defined and Pareto-efficient.

Remark 2: As a by-product of Proposition 1, for each veto vector $v$, each $>\in R$ and each $a \in \operatorname{VRP}_{v}(>)$, we can find a partition $\left(X_{1}, \ldots, X_{n}\right)$ of $A \backslash\{a\}$ such that

$$
X_{i} \subseteq L\left(a,>_{i}\right) \text { and } \bigcap_{i \in I} X_{i}=\emptyset \text { and }\left|X_{i}\right|=v_{i}
$$

As a final result of this section, we show that no selection of a VRP rule is Maskin monotonic. This implies that one cannot implement a selection of it by focusing on simultaneous mechanisms.

Proposition 2. For each veto vector $v$, if $f \subseteq V R P_{v}$ and $f$ is Maskin monotonic, then $f=V R P_{v}$.

Proof. Take some $f \subseteq \mathrm{VRP}_{v}$. If some voter has no vetoes then she plays no role in the outcome. On the contrary, if some voter has $k-1$ vetoes then she is a dictator. Hence in the sequel we consider w.l.o.g. a veto vector $v$ such that $0<v_{i}<k-1 \forall i \in I$. For each $a \in A$ and 
each vector $v$, define $B_{v}^{a}=\left\{>\in R \mid l\left(a,>_{i}\right)=v_{i} \forall i \in I\right.$ and $\left.l(a,>)=k-1\right\}$. Take some $>\in B_{v}^{a}$. It follows that $L\left(a,>_{i}\right) \cap L\left(a,>_{j}\right)=\emptyset$ for any $i, j \in I$ and $\{a\} \in \operatorname{VRP}_{v}(>)$. In order to prove that $f(>)=\{a\}$, assume that there is some $y \neq a$ and $y \in f(>)$. In this case, $l\left(y,>_{i}\right) \geq v_{i}$ for each $i \in I$, which implies that $y$ Pareto dominates $a$, a contradiction.

Since preferences over alternatives are unrestricted, for any partition $\left(X_{1}, \ldots, X_{n}\right)$ of $A \backslash\{a\}$ with $\left|X_{i}\right|=v_{i} \forall i \in I$, there is some profile $>\in B_{v}^{a}$ such that $L\left(a,>_{i}\right)=X_{i} \forall i \in I$. Consider any profile $>^{\prime} \in R$ with $\{a\} \in \operatorname{VPR}_{v}\left(>^{\prime}\right)$. By Remark 2, there exists a profile $>\in B_{v}^{a}$ with $L(a,>) \subseteq$ $L\left(a,>^{\prime}\right)$ and $a \in f(>)$. Then by Maskin Monotonicity $a \in f\left(>^{\prime}\right)$, as required.

\subsection{Simultaneous veto mechanisms}

In a simultaneous veto mechanism $\mathrm{SV}_{v}$, the strategy space of voter $i$ is denoted $M_{i}=\{X \subseteq$ $\left.A|| X \mid=v_{i}\right\}$, where $v_{i}$ denotes the number of alternatives voter $i$ vetoes. In the mechanism $\mathrm{SV}_{v}$, we let $v$ denote the veto vector that satisfies (1); this implies that at least some alternative remains non-vetoed for any message profile. The class of SV mechanisms is broad. It includes for instance the mechanism in which the veto rights are equally split among voters (i.e. $v=(1,1, \ldots, 1)$ when $k=n+1)$ so that all voters have the right of vetoing exactly one alternative. This class also includes dictatorships: when a voter concentrates all the veto rights (i.e. $v=(k-1,0, \ldots, 0)$ or a permutation of it). The rest of SV mechanisms allow some inequality on the veto rights of the different voters, even though, for any combination of voters and alternatives, there is a simultaneous veto mechanism with almost fair distribution of vetoes: $\left|v_{i}-v_{j}\right| \leq 1$ for any pair $i, j$ of voters.

Definition 1. The simultaneous veto mechanism $S V_{v}: M \equiv \prod_{i \in I} M_{i} \rightarrow \Delta$ with veto vector $v=$ $\left(v_{1}, \ldots, v_{n}\right)$ associates each message profile $m \in M$ to the lottery

$$
S V_{v}(m)=\operatorname{unif}\left(A \backslash \bigcup_{i \in I} m_{i}\right)
$$

Note that an SV mechanism selects uniformly an alternative among the non-vetoed ones.

If in the profile $m \in M, a \in m_{i}$ for some $i \in I$, then alternative $a$ is vetoed in $m$, otherwise $a$ is non-vetoed. For each simultaneous veto mechanism $\mathrm{SV}_{v}$, each voter $i$ and each $m_{-i} \in$ $M_{-i}$, let $V\left(m_{-i}\right)=\left\{a \in A \mid a \in m_{j}\right.$ for some $\left.j \neq i\right\}$ denote the set of vetoed alternatives by the opponents of voter $i$. Similarly, $N V\left(m_{-i}\right)=\left\{a \in A \mid a \notin V\left(m_{-i}\right)\right\}$ stands for the set of nonvetoed alternatives in $m_{-i}$. 
It is rather intuitive that SD implies that, in any simultaneous veto mechanism, voter $i$ 's best response is to veto her $v_{i}$ least preferred alternatives among those in $N V\left(m_{-i}\right)$ as stated by the next result. This in turn implies that each simultaneous veto mechanism $\mathrm{SV}_{v}$ is $\mathrm{DE}$ as stated by the next result.

Proposition 3. Assume that the domain $\kappa(>)$ satisfies $S D$. For any preference profile $>\in R$ and any simultaneous veto mechanism $S V_{v}$ :

1. the best response of a voter with preferences $>_{i}$ is to veto her $v_{i}$ least preferred alternatives among $N V\left(m_{-i}\right)$, that is:

$$
\forall \geq_{i}^{*} \in \kappa\left(>_{i}\right), \quad B R\left(\geq_{i}^{*}, m_{-i}\right)=\left\{a \in N V\left(m_{-i}\right)|| L\left(a,>_{i}\right) \cap N V\left(m_{-i}\right) \mid<v_{i}\right\}
$$

2. the outcome is deterministic in any equilibrium so that $S V_{v}(m) \in A$ for any $m \in N^{S V_{v}}\left(\geq^{*}\right)$ and any $\geq^{*} \in \kappa(>)$.

Proof. 1. For any simultaneous veto mechanism, the attainable set $g\left(M_{i}, m_{-i}\right)$ for voter $i$ contains any uniform lottery with support in $N V\left(m_{-i}\right)$. For any $m_{-i} \in M_{-i}$, we denote by $r$ the cardinal of $N V\left(m_{-i}\right)$ alternatives with $r \geq k-\sum_{j \in I \backslash\{i\}} v_{j}=v_{i}+1$. Then since $\geq_{i}^{*}$ satisfies SD, the most preferred outcome in $g\left(M_{i}, m_{-i}\right)$ for voter $i$ is the uniform lottery among her most preferred $r-v_{i}$ alternatives in $N V\left(m_{-i}\right)$. Thus, the best response of voter $i$ is to veto her $v_{i}$ least preferred alternatives in $N V\left(m_{-i}\right)$.

2. Fix some simultaneous veto mechanism $\mathrm{SV}_{v}$ and consider any equilibrium $m \in N^{\mathrm{SV}_{v}}\left(\geq^{*}\right)$. Assume by contradiction that $\mathrm{SV}_{v}(m) \notin A$ so that $\operatorname{supp}\left(\mathrm{SV}_{v}(m)\right) \geq 2$. This, in turn, implies that there are at least two voters, denoted $i$ and $j$, whose choices of vetoes intersect, $m_{i} \cap m_{j} \neq \emptyset$. However, as stated by 1 ., the best response of voter $i$ for any $m_{-i}$, denoted $B R\left(\geq_{i}^{*}, m_{-i}\right)$, is included in $N V\left(m_{-i}\right)$. Thus, in equilibrium, $B R\left(\geq_{i}^{*}, m_{-i}\right) \cap m_{j}=\emptyset$ for any voter $j \neq i$ which contradicts that $m$ is an equilibrium as desired.

Proposition 3 implies that, in equilibrium, a voter only vetoes alternatives that she prefers less than the outcome. Moreover, one can check that the converse also holds: each profile in which every voter $i$ vetoes $v_{i}$ unique alternatives while she prefers the outcome to each of these $v_{i}$ alternatives is an equilibrium, which gives a first characterization of the set of equilibria of simultaneous veto mechanisms. More formally, for each simultaneous veto mechanism $\mathrm{SV}_{v}$ and each utility profile $>$, we have: 


$$
N^{\mathrm{SV}_{v}}\left(\geq^{*}\right)=\left\{m \in M \mid \mathrm{SV}_{v}(m)=a \text { with } a>_{i} x \text { for each } i \in I \text { and } x \in m_{i}\right\}
$$

Yet, the characterization provided by (4) is silent on the alternatives that are selected in equilibrium. It turns out that the implemented alternatives consists of the $\mathrm{VRP}_{v}$ outcomes as discussed in the next section.

\subsection{Implementation of the Veto by random priority rules}

The next result states that the rule $\mathrm{VRP}_{v}$ coincides with the set of equilibrium outcomes of the simultaneous veto mechanism $\mathrm{SV}_{v}$. Before stating the main theorem, a key element of the result is that any equilibrium outcome is Pareto-efficient as stated by the next lemma.

Lemma 1. Assume that the domain $\kappa(>)$ satisfies $S D$ and take an arbitrary veto vector $v$ satisfying (1). For any $>\in R$ and any $\geq^{*} \in \kappa(>)$ and any $m \in N^{S V_{v}}\left(\geq^{*}\right), S V_{v}(m)$ is Pareto-efficient.

Proof. Consider some equilibrium profile $m$ and let $x \in A$ be its outcome (as shown by Proposition 3, for any veto vector $v$, the $\mathrm{SV}_{v}$ mechanism is DE). If $x$ is Pareto inefficient, there is some $y$ with $y>_{i} x$ for any voter $i \in N$ while $y$ is vetoed by some voter $j$. However, $m$ cannot be an equilibrium since $j$ has a profitable deviation. Indeed, consider the strategy $m_{j}^{\prime}$ in which voter $j$ vetoes $x$, does not veto $y$ while keeping vetoed the remaining alternative in $m_{j}$ (if any). Hence, $\operatorname{SV}_{v}\left(m_{j}^{\prime}, m_{-j}\right)=\{y\}$ and $y>_{j} x$ which shows that $m_{j}^{\prime}$ is a profitable deviation. Thus, any equilibrium outcome of the $\mathrm{SV}_{v}$ mechanism is Pareto-efficient.

Example (Condorcet Cycle): Let $I=\{1,2,3\}$ and $A=\{a, b, c, d\}$ respectively denote the set of voters and alternatives with preferences represented by Figure 1. The four alternatives form a Condorcet cycle with alternative $d$ being ranked third for every voter. Assuming that the veto vector coincides with $v=(1,1,1)$, the mechanism $S V_{v}$ admits a unique equilibrium outcome $d$ in this profile, since it is the only alternative not ranked last. Now, if we shift $d$ in the voters' rankings so that $d$ is ranked second or first for every voter, $d$ remains the only equilibrium outcome. If, on the contrary, $d$ is demoted to the last position of every voter, then $a, b$ and $c$ can be selected in some equilibrium. This example characterizes one property of these mechanisms: the priority is given to remove the least preferred alternatives of each voter.

The next Theorem presents one of the main results of this work, that is that SV achieve the Nash implementation of a Pareto efficient $S C R$. 


\begin{tabular}{c|c|c}
$>_{1}$ & $>_{2}$ & $>_{3}$ \\
\hline$a$ & $c$ & $b$ \\
$b$ & $a$ & $c$ \\
$\mathbf{d}$ & $\mathbf{d}$ & $\mathbf{d}$ \\
$c$ & $b$ & $a$
\end{tabular}

(a) Preference Profile
Simultaneous veto

$\cdot c$ is vetoed in any equilibrium (worst for 1 )

$\cdot b$ is vetoed in any equilibrium (worst for 2 )

$\cdot a$ is vetoed in any equilibrium (worst for 3 )

$\cdot d$ unique winner in equilibrium

(b) Pareto efficiency of SV mechanisms

Figure 1: Condorcet Cycle and SV mechanisms

Theorem 1. Assume that the domain $\kappa(>)$ satisfies SD. For each veto vector $v$, the simultaneous veto mechanism $S V_{v}$ Nash implements the correspondence $V R P_{v}$, so that

$$
\{a\}=S V_{v}(m) \text { for some } m \in N^{S V_{v}}\left(\geq^{*}\right) \Longleftrightarrow\{a\} \in V P R_{v}(>)
$$

Proof. In order to see that any equilibrium outcome of an SV mechanism is part of the VRP rule, take some preference profile $>\in R$. Proposition 3 ensures that $\mathrm{SV}_{v}$ is $\mathrm{DE}$ so that no pair of voters vetoes common alternatives. Assume by contradiction that there is some $a \in A$ such that $\{a\} \notin \operatorname{VPR}_{v}(>)$ whereas $\operatorname{SV}_{v}(m)=\{a\}$ for some $m \in N^{\operatorname{SV}_{v}}\left(\geq^{*}\right)$. That means that there is some coalition $J$ for which $l\left(a,>_{J}\right)<v_{J}$. Since no pair of voters vetoes common alternatives, $l\left(a,>_{J}\right)<v_{J}$ is only possible if some voter $j \in J$ is vetoing some alternative $a^{\prime} \in A$ such that $a^{\prime}>_{j} a$. This means that $j$ is not playing her best response, so that $m$ is not a Nash equilibrium, a contradiction.

In order to understand that for any veto vector $v$ and any $>\in R$ and any alternative in $a \in \operatorname{VRP}_{v}(>)$, there is an equilibrium of the $\mathrm{SV}_{v}$ mechanism that selects it, it suffices to apply Remark 2. Indeed, Remark 2 states for each veto vector $v$, each $>\in R$ and each $a \in \operatorname{VRP}_{v}(>)$, we can find a partition $\left(X_{1}, \ldots, X_{n}\right)$ of $A \backslash\{a\}$ such that $X_{i} \subseteq L\left(a,>_{i}\right)$ and $\bigcap_{i \in I} X_{i}=\emptyset$ and $\left|X_{i}\right|=v_{i}$. Hence, consider the profile $m$ with $m_{i}=X_{i}$ so that $\operatorname{SV}_{v}(m)=\{a\}$. Since $m_{i} \subseteq L\left(a,>_{i}\right)$, each voter $i$ is playing a best response to $m_{-i}$, which concludes the proof.

\subsection{Coalitional implementation via simultaneous vetoes}

The next result shows that the Nash implementation via SV mechanisms depicted by Theorem 1 is robust to the introduction of alternate ways of communication. Indeed, in a Nash equilibrium, no communication is assumed to arise before the strategies are chosen. If communication takes place before the vote, then it might be the case that the notion of Nash equilibrium is not well adapted. Hence, we focus on coalition-proof equilibria in which 
coalitions can deviate. Yet, in contrast with the strong equilibrium notion, coalitional deviations are only allowed when they are self-enforcing, that is no sub-coalition can in turn deviate after the coalitional deviation and obtain a profitable deviation. As we now show, any Nash equilibrium of a SV mechanism is coalition-proof.

Proposition 4. Assume that the domain $\kappa(>)$ satisfies SD. For each veto vector $v$, the simultaneous veto mechanism $S V_{v}$ coalitionally implements the rule $V R P_{v}$.

The intuition for this result is related to the equilibrium strategies. Indeed, as described by Proposition 3, every voter vetoes only alternatives that she deems less preferred than the equilibrium outcome and no pair of voters vetoes common alternatives. Therefore, there is no joint coalition than can benefit all voters in the coalition, which leads to coalitionproofness.

Proof. Theorem 1 proves that each $\mathrm{SV}_{v}$ Nash implements the rule $\mathrm{VRP}_{v}$. It then suffices to prove that every Nash equilibrium of $\mathrm{SV}_{v}$ is coalition-proof. In order to do so, take some preference profile $>\in R$ and assume that the domain $\kappa(>)$ satisfies SD. Consider some equilibrium $m \in N^{\mathrm{SV}_{v}}\left(\geq^{*}\right)$ with $\mathrm{SV}_{v}(m)=\{a\}$. Since (1) holds and any equilibrium is deterministic, exactly $k-1$ alternatives are vetoed in the profile $m$ so that each alternative is vetoed by exactly one voter.

First we show that self-enforcing deviations from a Nash equilibrium only lead to pure alternatives. Assume by contradiction that some coalition $J \in C^{I}$ has some self-enforcing deviation $m_{J}^{\prime}$ with $\left|\operatorname{supp}\left(\mathrm{SV}_{v}\left(m_{J}^{\prime}, m_{-J}\right)\right)\right|>1$. In the profile $\left(m_{J}^{\prime}, m_{-J}\right)$, there is some voter $j^{*}$ in $J$ that vetoes the same alternative as some other voter since in the profile $m$ each alternative is vetoed by exactly one voter. Since voter $j^{*}$ has strict preferences over the alternatives, voter $j^{*}$ has a profitable deviation in the profile $\left(m_{J}^{\prime}, m_{-J}\right)$ : vetoing her $v_{j^{*}}$ least preferred alternatives in $N V\left(m_{-j^{*}}\right)$. This shows that coalitional deviations leading to lotteries are not self-enforcing. Hence, only deviations which lead to a deterministic outcome are self-enforcing.

Assume that the equilibrium $m$ is not coalition-proof. This implies that some coalition $J \in C^{I}$ has a self-enforcing deviation $m_{J}^{\prime}$. Since $m_{J}^{\prime}$ is self-enforcing, as previously shown, it must be that $\mathrm{SV}_{v}\left(m_{J}^{\prime}, m_{-J}\right)=\{b\}$ for some $b \in A$. Since $m_{J}^{\prime}$ is self-enforcing, $b>_{j} a$ for all $j \in J$. Since coalition $J$ can reach $b$ as an outcome it must be the case that under $m, b$ is vetoed by some voter $j^{\prime} \in J$. But this contradicts $m$ being a Nash equilibrium since every voter only vetoes alternatives that she prefers less than $a$ in $m$ (see Proposition 3) whereas $a>_{j} b$ for any $j \in J$, concluding the proof. 


\section{Vetoes and Coalitional Implementation}

In this section, we characterize rules that can be coalitionally implementated (Theorem 2). We finally show that coalitional implementation of a Pareto efficient rule is not possible with deterministic mechanisms.

\subsection{Coalitional implementation}

Up to this point, this work has shown that the $\mathrm{VRP}_{v}$ are coalitionally implementable via the SV mechanisms. This section considers the reverse questions: which are the Pareto SCRs that can be coalitionally implemented? How are the mechanisms that achieve this implementation? As we show in the sequel, under some conditions, the idea of veto is closely related to the one of coalitional implementation.

To the best of our knowledge, if one excludes integer/modulo games and dictatorships, few mechanisms in the literature have been shown to implement a Pareto efficient rule. Among them, a salient class are the Kingmaker games (see Maskin [1999] and Hurwicz and Schmeidler [1978]) where one voter decides the identity of the voter who will, in turn, decide the outcome. As we now show, these mechanisms do not achieve coalitional implementation. Example (Kingmaker game): Let $A=\left\{a_{1}, a_{2}, a_{3}, a_{4}\right\}$ and $N=\{1,2,3\}$ respectively denote the set of alternatives and the set of voters. The strategy sets of voters 1, 2 and 3 are denoted by $S_{1}=\{2,3\}$ and $S_{2}=S_{3}=A$. Define $g: S_{1} \times S_{2} \times S_{3}$ with $g^{K M}\left(s_{1}, s_{2}, s_{3}\right)=a_{s_{1}}$. In other words, the winning alternative is the alternative announced by the voter nominated by voter 1. It can be shown that the mechanism $g^{K M}$ Nash implements the SCR $f(>)=\left\{a \in A \mid a>_{j}\right.$ $b$ for some $j \in\{2,3\}\}$ while $f$ is Pareto-efficient. Yet, this mechanism does not coalitionally implement $f$. Indeed, observe that any profile $s$ with $s_{2}=s_{3}=a$ is an equilibrium for any preference profile as long as the nominated voter, say 3, ranks a first in her preferences. Indeed, voter 3 is best responding whereas neither the non-nominated voter, voter 2 , nor voter 1 can alter the outcome. However, if both 1 and 2 prefer $b$ to $a$, they have a coalitional deviation that leads to $a$ (i.e. $s_{1}=2$ and $s_{2}=b$ ) which shows that this mechanism admits equilibria which are not coalition-proof.

In order to derive conditions on coalitionally implementable rules, we impose the completeness of the preference extensions over lotteries. Observe that in the previous sections we did not require that the preferences over lotteries were complete. This is so due to the 
structure of SV mechanisms. Indeed, they are constructed in such way that for each voter $i \in I$ for any profile $m_{-i}$ there is an element of $\mathrm{SV}_{v}\left(M_{i}, m_{-i}\right)$ which voter $i$ strictly prefers to any other element of the attainable set under SD. The existence of a maximal element in the attainable set does not require that the voter can compare each pair of elements in the attainable set. Yet, since we do not know the structure of attainable sets in an arbitrary mechanism, we assume that the implementing mechanisms satisfy completeness, which definition is as follows.

Definition 2. For each $i \in N$ and each $>_{i} \in R_{i}$, the set $\kappa\left(>_{i}\right)$ of admissible preference extensions satisfies completeness if for each $\geq_{i}^{*} \in \kappa\left(>_{i}\right)$ for any $\alpha, \beta \in \Delta$, either $\alpha \geq_{i}^{*} \beta$ or $\beta \geq_{i}^{*} \alpha$. The domain $\kappa(>)$ satisfies completeness if each $\kappa\left(>_{i}\right)$ is complete for each $i \in N$ and each $>_{i} \in R_{i}$.

For each mechanism $g$, $\operatorname{veto}(g, J)$ represents the sets of alternatives that coalition $J$ can exclude from being chosen for any strategy of her opponents. Formally, for each coalition $J \in C^{I}$,

$$
\operatorname{veto}(g, J)=\left\{X \subseteq A \mid \exists m_{J} \in M_{J} \text { s.t. } X \cap \operatorname{supp}\left(g\left(m_{J}, m_{-J}\right)\right)=\emptyset \forall m_{-J} \in M_{-J}\right\} .
$$

A mechanism $g$ is neutral-on-its-vetoes for coalition $J$ if and only if $X \in \operatorname{veto}(g, J)$ for some $X \subseteq A$, then any set $Y$ with $|Y|=|X|$ satisfies $Y \in \operatorname{veto}(g, J)$. A mechanism $g$ is neutralon-its-vetoes if $g$ is neutral-on-its-vetoes for every coalition $J \in C^{I}$. We write $v_{g}(J)$ to denote the veto power of coalition $J$ under the mechanism $g$ when $g$ is neutral-on-its-vetoes.

The main result of this section is as follows.

Theorem 2. Assume that the domain $\kappa(>)$ satisfies completeness, SD and PREX. Let $f$ be a Pareto-efficient SCR. If $f$ is coalitionally implementable by some $\mathbf{D E}$-mechanism $g$, then :

1. $g$ is neutral-on-its-vetoes,

2. for any partition $(J, K)$ of $I, v_{g}(J)+v_{g}(K)=k-1$,

3. if $a \in f(>)$, then $l\left(a,>_{i}\right) \geq v_{g}(i)$ for each $i \in I$.

This theorem gives several insights regarding coalitionally implementable SCRs.

The fact that any implementing mechanism $g$ is neutral-on-its-vetoes is noteworthy. It shows that no mechanism where some voter(s) has decisive power over a subset of alternatives are ruled out (such as local dictatorships, see Börgers and Li [2019] for an analysis). 
Indeed, the neutrality of the vetoes combined with the partition property hints at the following conclusion: a mechanism that specifies the veto rights for each coalition is unavoidable to achieve coalitional implementation. Indeed, Theorem 2 implies that for any partition $(J, K)$ of the voters, either one of the coalitions can impose the outcome or both coalitions have positive veto power. These veto rights have implications on the rules one can expect to coalitionally implement. The property 3 shows that each voter is ensured to obtain an outcome which is not among his worst $v_{g}(i)$ alternatives. This means that any coalitionally implementable rule which fails to coincide with a $\mathrm{VRP}_{v}$ rule exhibits the following property: there is some coalition $J$ and some $a \in f(>)$ such that $l\left(a,>_{J}\right)<v_{g}(J)$. This implies an inefficiency of the rule since the voters in $J$ fail to coordinate their vetoes to ensure a collectively preferred alternative.

The next condition ensures that merging coalitions does not allocate supplementary veto power to the voters. This condition, No Gains from Cooperation, is satisfied by the SV mechanisms. In a mechanism satisfying NGC, the veto rights of a coalition only depend on the voting rights of each of the voters in the coalition, whereas this need not be the case when this condition fails.

Definition 3. A mechanism $g$ satisfies No Gains from Cooperation (NGC) if for all $J, K \subset I$ such that $J \cap K=\emptyset$, for any $X \in \operatorname{veto}(g, J \cup K)$ there exist $Y \in \operatorname{veto}(g, J)$ and $Z \in \operatorname{Veto}(g, K)$ such that $X=Y \cup Z$.

Proposition 5. Under the assumptions of Theorem 2, let $f$ be a coalitionally implementable SCR via a mechanism $g$. The following claims hold:

1. If $g$ satisfies $N G C$, then $f \supseteq V R P_{v}$ for some $v$.

2. If $k \geq n$, then at least some voter has positive veto power.

If one imposes the strong condition of NGC, Theorem 2 implies that the implemented rule $f$ selects all alternatives that some VRP rule prescribes. To see why it is true consider observe that Theorem 2 imposes that $v_{g}(J)+v_{g}(K)=k-1$ for any partition of the voters. Since NGC implies that the veto powers of the voters are additive, we can prove that the mechanism $g$ admits any of the equilibria of the $S V$ mechanisms, which implies the result.

Moreover, with at least more alternatives than voters, we can show that at least some voter has positive veto power. This means that any coalitionally implementable SCR violates the well-known condition of No-Veto power. This condition, extensively used in implementation theory, states that whenever $n-1$ voters rank the same alternative on top of 
their preferences, "the last voter cannot prevent the alternative from being $f$-optimal (i.e. "he cannot veto it")" as Maskin [1999] puts it. With less alternatives than voters, this need not be the case as we discuss in the next sections.

\subsection{Coalitional implementation with two alternatives: endogenous vetoes}

This section shows how to coalitionally implement the majority rule. The main difficulty here is to ensure that each equilibrium is coalition-proof while ensuring that no voter has veto power (if a voter has veto power, he is a dictator in this setting). Our results contrast with the results of Xiong [2021] that shows that, in this setting, no rule (except dictatorship) is implementable through a direct mechanism or through an indirect mechanism that satisfies some regularity condition. Note that our mechanism does not satisfy Xiong [2021]'s conditions. The main novelty of our mechanism is that it incorporates the coalition formation rules, whereas most mechanisms simply ask information regarding the different candidates.

Let $A=\{a, b\}$ be the set of alternatives and $n=2 p+1$ be the number of voters to ensure that for each profile $>, \operatorname{MR}(>)=\left\{a \in A||\left\{i \in I \mid a>_{i} b\right\}|>|\left\{i \in I \mid a>_{i} b\right\} \mid\right\}$ defines the unique

majority preferred alternative. We let $g^{P V}$ denote the partial vetoes mechanism such that the strategy $m_{i}$ of each voter equals $m_{i}=\left(n_{i}, c_{i}\right) \in M^{i}: \mathcal{I}^{p} \backslash\{i\} \times A$ so each voter $i$ nominates a group of $p$ voters excluding himself and chooses an alternative. For each $m \in M$, let $N(m)=$ $\left\{i \in N \mid i=n_{j}\right.$ for some $\left.j \in N\right\}$ denote the set of nominated voters and $C(m)=\left\{a \in A \mid a=c_{i}\right.$ for some $i \in N(m)\}$ denote the set of chosen alternatives by some nominated voter. For each $m \in M$, the outcome of the partial veto mechanism $g^{P V}$ is as follows.

Let $M$ be a majority of voters with $|M|=p+1$. In the profile $m$, the strategies of the voters in $M$ form a block against $x$ if for each $i \in M$ the strategy $s_{i}$ satisfies:

1. for each $i \in M, n_{i}=M \backslash\{i\}$

2. for each $i \in M, c_{i}=x$.

The voters in $M$ form a block against $x$ if they all nominate each other while choosing $x$. If in the profile $m$, there is a majority who forms a block against $x$, the outcome is $y$. Otherwise, the outcome $g^{P V}(m)$ is lottery $\beta$ with: 


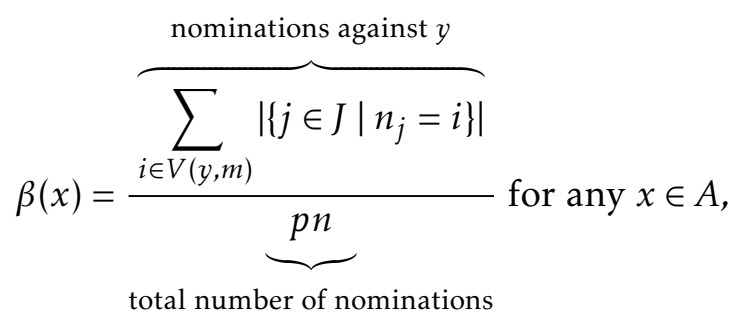

where $V(y, m)=\left\{i \in N(m) \mid c_{i}=y\right\}$ denotes the nominated voters who cast a veto against $y$ with $y \neq x$ and $\left|\left\{j \in J \mid n_{j}=i\right\}\right|$ is the score of voter $i$ at profile $m$, i.e. the number of voters who nominate $i$. This means that the probability that $x$ is selected equals the frequency with which $x$ is not announced by the nominated voters.

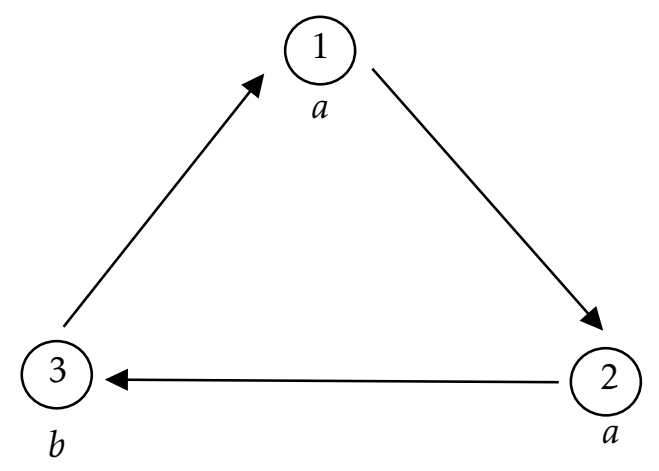

$a$ with prob $1 / 3$ and $b$ with prob $2 / 3$

Figure 1.a: No block

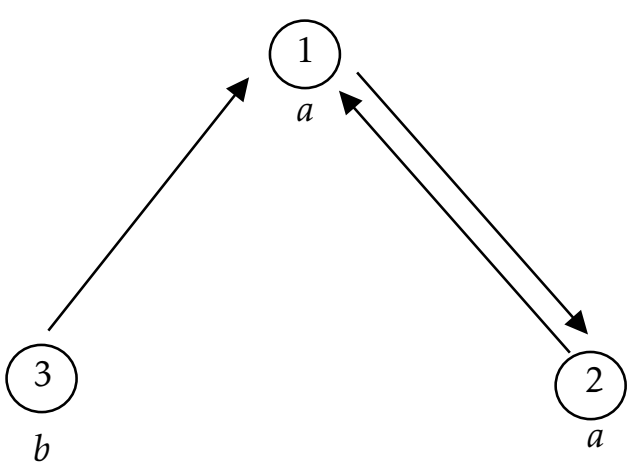

Block of 1,2 against $a: b$ is elected

Figure 1.b: Block of voters

Figure 1: A mechanism with partial vetoes

Figure 1 represents two instances of the mechanism with three voters (i.e. when $p=1$ ). In the figure, the arrows represent the nominations whereas the letters denote the alternatives chosen by the voter. In Figure 1.a, the profile $m$ is depicted with $m_{1}=(2, a), m_{2}=(3, a)$ and $m_{3}=(1, b)$. This leads to a lottery where $a$ is selected with probability $1 / 3$ and $b$ with probability $2 / 3$ since $a$ is chosen by both 1 and 2 (both are nominated) and $b$ is chosen by 3 (nominated by 2). Figure 1.b presents the profile $m^{\prime}$ with a block of voters with $m_{1}^{\prime}=(2, a)$, $m_{2}^{\prime}=(1, a)$ and $m_{3}^{\prime}=(1, b)$. In this case, 1 and 2 form a block against $a$ and alternative $b$ is selected. 
At any profile in which no block is formed, any nominated voter strictly prefers to announce her least preferred alternative since this will strictly decrease the probability that this alternative wins. Observe that in the mechanism $g^{P V}$, every voter and every coalition with less than $p+1$ voters has zero veto power whereas any coalition with at least $p+1$ voters has a veto power of 1 .

Theorem 3. Let $A=\{a, b\}$. Assume that the domain $\kappa(>)$ satisfies SD. The partial vetoes mechanism $g^{P V}$ coalitionally implements the majority rule $M R(>)$.

Proof. W.l.o.g. assume that any voter $i$ in $\{1, \ldots, p+1\}$ is such that $a>_{i} b$ so that $a$ is the majority preferred alternative. Observe that the profile $m$ where every voter in $\{1, \ldots, p+1\}$ nominate a subset of $\{1, \ldots, p+1\}$ and every such voter chooses $b$ is an equilibrium that selects $a$ since it creates a block against $b$. No block of voters against $a$ can be an equilibrium since there at most $p$ voters who prefer $b$ to $a$.

To see that $a$ is the unique equilibrium outcome, consider the two following cases.

First, if all voters prefer $a$ to $b$, the claim is immediate, since whenever a voter is nominated he chooses $b$, so that only $a$ can be elected at equilibrium.

Assume now that this is not the case, so that there is some voter, say $h$, with $b>_{h} a$.

We first claim that, in equilibrium, any voter $i$ with $a>_{i} b$ announces $b$. This is immediate if the voter $i$ is nominated since this decreases the probability that $b$ is elected. Assume, by contradiction, that $i$ is not nominated in some equilibrium $m$ while choosing $a$. The voter $h$, with $b>_{h} a$, nominates $p$ voters by definition. Since there are at most $p$ voters (including voter $h$ ) who prefer $b$ to $a$, this means that $h$ nominates some voter, say $j$, with $a>_{j} b$. Yet, in equilibrium, $j$ announces $b$. This means that voter $h$ prefers to deviate and nominate $i$ (who chooses $a$ ) since this will strictly increase the probability that $b$ is elected, showing that $m$ is not an equilibrium.

Finally, it suffices to see that any voter who prefers $a$ to $b$ only nominates voters who prefer $a$ to $b$. Indeed, as previously argued, any voter who prefers $a$ to $b$ chooses $b$ in any equilibrium. Moreover, any voter who prefers $b$ to $a$ will choose $a$ if nominated. Thus, any voter who prefers $a$ to $b$ only nominates voters who prefer $a$ to $b$. Therefore, there is always a block against $b$, which shows that the unique equilibrium outcome is $a$, the majority preferred alternative.

This game describes a coalition formation game that implements the majority rule. An adaptation of the previous mechanism can be used to implement qualified majority rules 
with a status quo, where an alternative $a$ is elected if a sufficiently large majority prefers $a$ to $b$ and otherwise $b$ is implemented.

\subsection{Coalitional implementation without NGC}

In this section, we present an example of a mechanism that coalitionally implements a SCR with three alternatives and voters where only one of the voters has veto power. While we consider the case with $n=k=3$ for ease of exposition, similar mechanisms can be designed where the sum of the individuals vetoes equals $k-2$. These mechanisms are more complex than the simultaneous veto mechanisms. This is unsurprising given that these mechanisms violate NGC and hence the voters need to coordinate their actions to ensure an effective veto.

Letting $\mathcal{A}^{2}$ denote the sets of two different alternatives, we define the mechanism $g: M \rightarrow$ $\Delta$ with:

- $M_{1}=\mathcal{A}^{2} \times\{2,3\}$ with a generic element being $m_{1}=\left(v^{1}, c^{1}, n^{1}\right)$.

- $M_{2}=\mathcal{A}^{2} \times\{1,3\}$ with a generic element being $m_{2}=\left(c_{1}^{2}, c_{2}^{2}, n^{2}\right)$.

- $M_{3}=\mathcal{A}^{2} \times\{1,2\}$ with a generic element being $m_{3}=\left(c_{1}^{3}, c_{2}^{3}, n^{3}\right)$.

Thus, voter 1 vetoes 1 alternative, announces 1 different alternative and nominates a voter. Voters 2 and 3 announce two alternatives and nominate a voter. Let $N(m)=\{i \in I \mid$ $\exists j \in I$ s.t. $\left.n^{j}=\{i\}\right\}$ be the set of nominated voters in the profile $m$ and $C(m)=\bigcup_{i \in N(m)} c^{i}$ be the set of alternatives announced by nominated voters. The outcome is a lottery $\beta$ with the support being $C(m) \backslash v^{1}$, the alternatives not vetoed by voter 1 . The weights in the lottery are determined by frequencies. So if $a \in C(m) \backslash v^{1}$ then:

$$
\beta(a)=\frac{\sum_{i \in N(m)} 1_{\left\{a \in c^{i}\right\}}}{\sum_{b \in C(m) \backslash v^{1}}\left[\sum_{i \in N(m)} 1_{\left\{b \in c^{i}\right\}}\right]} .
$$

Thus, the probability of $a$ in the resulting lottery is equal to the share of $a$ in the alternatives announced by nominated voters (excluding the alternative vetoed by voter 1 ).

This mechanism combines the SV mechanisms with the partial vetoes mechanism used in the setting with two alternatives. Indeed, once the alternative vetoed by player 1 is excluded, the voters behave in equilibrium as if they were playing the partial vetoes mechanism over the two non-vetoed alternatives. This ensures that every equilibrium is coalition-proof.

Proposition 6. Assume that the domain $\kappa(>)$ satisfies SD. The mechanism g coalitionally imple- 


\begin{tabular}{c|c|c}
$>_{1}$ & $>_{2}$ & $>_{3}$ \\
\hline $\mathbf{a}$ & $d$ & $d$ \\
$b$ & $\mathbf{a}$ & $\mathbf{a}$ \\
$c$ & $b$ & $b$ \\
$d$ & $c$ & $c$
\end{tabular}

(a) Preference Profile
Simultaneous veto

$\cdot d$ is vetoed in any equilibrium (worst for 1 )

$\cdot c$ is vetoed in any equilibrium (worst for 2 and 3 )

- $b$ is vetoed by either 2 or 3 in any equilibrium

- $a$ unique winner in equilibrium

(b) Pareto efficiency of SV mechanisms

Figure 2: An example of an SV mechanism

ments the SCR $f$ defined as follows:

$$
a \in f(>) \Leftrightarrow l\left(a,>_{J}\right) \geq v(J) \quad \forall J \in C^{I}
$$

where $v(1)=1, v(2)=v(3)=0, v(1 \cup 2)=v(1 \cup 3)=2, v(2 \cup 3)=1, v(I)=2$.

\subsection{Coalitional implementation via deterministic mechanisms}

This section that coalitional implementation via deterministic mechanisms is severely restricted. We first show with an example that veto-based mechanisms with deterministic tie-breaking rules tend to generate Pareto dominated equilibria. We then provide some sufficient conditions that ensure that the only coalitionally implementable rule with deterministic mechanisms is dictatorship.

Example (Pareto inefficiency of deterministic mechanisms): Let $I=\{1,2,3\}$ and $A=\{a, b, c, d\}$ respectively denote the set of voters and the set of alternatives. Assume that the the preferences of the voters are the ones presented in Figure 2. In the profile each column corresponds to a voter, such that the first column represents the preferences of voter 1 , the second column represents the preferences of voter 2 , and so on. The alternatives are placed from those most preferred in the first row to those least preferred in the last row.

Veto-rank mechanism. Each voter selects one alternative to veto and then ranks the remaining alternatives. The alternative ranked first receives a score of 2 , the alternative ranked second receives a score of 1 and the alternative ranked third gets a score of 0 . The veto-rank mechanism then selects the alternative with the highest sum of the voters scores from the nonvetoed alternatives, with ties being broken alphabetically. Assume that voter 1 vetoes $d$ while voters 2 and 3 both veto $a$. Observe that any profile with these vetoes that leads to the election of $b$ is a Nash equilibrium. Indeed, in this case $b$ is the best alternative for each voter from among the non-vetoed alternatives. Therefore, no player has a profitable unilat- 
eral deviation so that $b$ is selected in equilibrium. However, $b$ is Pareto-dominated by $a$. In this case voters' aggressive behavior, vetoing their opponent's top alternative, leads them to an inefficient equilibrium.

$S V$ mechanism. Letting $v=(1,1,1)$ denote the veto vector, it suffices to see that $a$ is the unique outcome of the SV mechanism in this preference profile (see Figure 1(b)). Observe that $d$ cannot be an equilibrium outcome since $d$ is the least preferred alternative for voter 1 and she can always prevent its election by vetoing it. A similar idea applies to $c$ since $c$ is the least preferred alternative of voters 2 and 3. Finally, $b$ cannot be an equilibrium outcome either. Indeed, observe that in equilibrium, no voter vetoes the same alternative as that vetoed by an opponent since she always benefits from vetoing the worst nonvetoed alternative. Thus, since in equilibrium some voter vetoes $c$, either voter 2 or 3 will also veto $b$, thereby preventing this alternative from winning.

We now prove that no deterministic mechanism which satisfies NGC can coalitionally implement a Pareto-efficient rule. This implies that lotteries off-equilibrium are key to obtain coordination among voters. It should be emphasized that other results in this direction exist in the literature. Indeed, Maskin [1978] considers implementation in Strong equilibria and Suh [1997] is concerned with double implementation in Nash and strong Nash equilibria. This is contrast with Moulin [1983] proves that any VRP rule is implementable in strong equilibria.

Theorem 4. Assume that $\kappa(>)$ satisfies SD and PREX. Let $f$ be a Pareto-efficient SCR by some deterministic mechanism $g$. If either $k \geq n$ or $g$ satisfies NGC, then $f$ is dictatorial.

The main logic of this impossibility result is rather simple. The notion of coalitional implementation imposes that either a voter is a dictator (veto power of $k-1$ ) or at least two voters have positive veto power. In the latter case, we prove that there exists Pareto dominated equilibria as for instance the example described in the introduction. This theorem proves that off-equilibrium lotteries are useful to avoid Pareto inefficiencies.

\section{Conclusion}

This paper contributes to the design of voting rules by proposing simple simultaneous mechanisms that endow voters with veto rights. These mechanisms, the SV mechanisms, ensure that every equilibrium outcome is Pareto efficient. Few mechanisms share this property in 
the literature, with the notable exception of the kingmaker mechanisms as proposed by Hurwicz and Schmeidler [1978]. Despite admitting a multiplicity of equilibria, the outcome of each equilibrium under a SV mechanism can be justified by a sequence of naive vetoes by different voters, showing that this multiplicity is better behaved than under the vast majority of rules. ${ }^{18}$

An important aspect of SV mechanisms is that each equilibrium is coalition-proof; in other words SV mechanisms achieve coalitional implementation. As we show, this notion of implementation is closely related to the introduction of veto rights in the implementing mechanism. With more alternatives than voters, at least some voter has veto rights in any mechanism that achieves coalitional implementation. With less alternatives than voters, it can be the case that only coalitions have veto rights; for instance, this is the case in the partial vetoes mechanism that implements the majority rule and in which coalitions are formed endogenously. Further work on the endogenous formation of coalition in voting environments seems to be a promising venue of research.

\section{A Technical Appendix}

It presents the proof of Theorem 2 that provides necessary conditions on the veto structure of a coalitionally implementable rule. It also shows the arguments behind the impossibility result of Theorem 4 and Proposition 5.

\section{A.1 Proof of Theorem 2}

The proof is divided in several lemmas. Lemma 2 establishes that the implementing mechanism endows the different coalitions with some veto power. Lemma 3 shows that a coalitionally implementable SCR must be neutral-on-its vetoes. Lemma 4 establishes the partition property. Finally, Lemma 5 states an important condition, the additive property, which establishes that the veto power of the different coalitions is additive.

Lemma 2. Assume that the preference domain $\kappa(>)$ satisfies completeness and PREX. If the mechanism $g$ coalitionally implements the SCR $f$, then, for any partition $(X, Y)$ of $A$ and any partition

\footnotetext{
${ }^{18}$ A natural application of our mechanisms is to use them as screening devices: namely, to use these mechanisms as a first step in a selection process. Indeed, it seems that removing alternatives that are deemed as undesirable by the different voters is a necessary first step for making a reasonable collective choice (see Manzini and Mariotti [2007] for a similar idea in decision theory and Horan and Sprumont [2020] for a two-stage collective choice procedure).
} 
$(J, H)$ of I either $Y \in \operatorname{vetO}(g, J)$ or $X \in \operatorname{veto}(g, H)$, but not both.

Proof. Let $(X, Y)$ be a partition of $A$ and $(J, H)$ be a partition of $I$. Consider the following preference profile $>$ and its extension $\geq^{*}$ (which exists due to PREX):

1. $\forall j, j^{\prime} \in J,>_{j}=>_{j^{\prime}}$ and $\forall h, h^{\prime} \in H,>_{h}=>_{h^{\prime}}$;

2. $\forall j \in J, \forall h \in H, \forall x \in X$ and $\forall y \in Y, x>_{j} y$ and $y>_{h} x$;

3. $\forall j, j^{\prime} \in J, \geq_{j}^{*}=z_{j^{\prime}}^{*}$ and $\forall h, h^{\prime} \in H, \geq_{h}^{*}=z_{h^{\prime}}^{*}$;

4. $\forall j \in J, \forall \beta, \gamma \in g(M)$ with $\sum_{x \in X} \beta(x)>0$ and $\sum_{x \in X} \gamma(x)=0, \beta>{ }_{j}^{*} \gamma$;

5. $\forall h \in H, \forall \beta, \gamma \in g(M)$ with $\sum_{y \in Y} \beta(y)>0$ and $\sum_{y \in Y} \gamma(y)=0, \beta>_{h}^{*} \gamma$.

Let $g$ be a mechanism that coalitionally implements $f$ so that there is some $m \in N^{g}(u)=$ $C^{g}(u)$ with $g(m)=\{a\}$ with some $a \in A$ with either $a \in X$ or $a \in Y$. Assume by contradiction that $Y \notin \operatorname{veto}(g, J)$ and $X \notin \operatorname{veto}(g, H)$.

- $a \in X$. Since $Y \notin \operatorname{veto}(g, J)$, there is some $m_{H}^{\prime} \in M_{H}$ such that $\operatorname{supp}\left(g\left(m_{J}, m_{H}^{\prime}\right)\right) \cap Y \neq \emptyset$. If $|H|=1$, hence $H=\{h\}$ for some $h$, then, by construction of $>$, the strategy $m_{H}^{\prime}$ is a profitable deviation for voter $h$, which contradicts $m$ being an equilibrium. If $|H|>1$, a similar logic applies. Since the preferences of all members of coalition $H$ coincide, there is a self-enforcing deviation for $H$ at $m$.

To see why observe first that for each voter $h \in H$ there exists $\widetilde{\beta} \in g\left(M_{H}, m_{J}\right)$ such that $\widetilde{\beta} \geq_{h}^{*} \gamma$ for all $\gamma \in g\left(M_{H}, m_{J}\right)$ (since $\geq^{*}$ is complete and $g(M)$ is finite by assumption). Since there is $m_{H}^{\prime} \in M_{H}$ such that $\operatorname{supp}\left(g\left(m_{J}, m_{H}^{\prime}\right)\right) \cap Y \neq \emptyset$, it follows $\operatorname{supp}(\widetilde{\beta}) \cap Y \neq \emptyset$ due to 4 . Denoting by $m_{H}^{\prime}$ the strategy such that $g\left(m_{J}, m_{H}^{\prime}\right)=\widetilde{\beta}$, observe that $m_{H}^{\prime}$ is selfenforcing for coalition $H$ since the preference extension of all members of coalition $H$ coincide and hence no coalition $H^{\prime} \subset H$ can achieve an outcome preferred to $\widetilde{\beta}$. This contradicts the fact that $m$ is a coalition-proof equilibrium.

- $a \in Y$. By the same logic, a similar contradiction arises.

Thus either $Y \in \operatorname{veto}(g, J)$ or $X \in \operatorname{veto}(g, H)$. Since the mechanism $g$ is well-defined, it is impossible that both conditions hold simultaneously, concluding the proof.

Lemma 3. Assume that the preference domain $\kappa(>)$ satisfies completeness, SD and PREX. If the mechanism g coalitionally implements a Pareto-efficient SCR $f$, then $g$ is neutral-on-its-vetoes. 
Proof. Consider some partition $(J, H)$ of $I$ and let $X \in \operatorname{veto}(g, J), x \in X$ and $x^{\prime} \in A \backslash X$. Thus, there exists $m_{J} \in M_{J}$ that vetoes $X$. Define the set $X^{\prime}=X \backslash\{x\} \cup\left\{x^{\prime}\right\}$ and observe that $|X|=\left|X^{\prime}\right|$. Write $Y=A \backslash\left(X \cup\left\{x^{\prime}\right\}\right)$, so that $A$ can be partitioned as follows:

$$
A=(X \backslash\{x\}) \cup\{x\} \cup\left\{x^{\prime}\right\} \cup Y=X^{\prime} \cup\{x\} \cup Y .
$$

Suppose, by contradiction, that $X^{\prime} \notin \operatorname{veto}(g, J)$. Lemma 2 implies that $Y \cup\{x\} \in \operatorname{veto}(g, H)$. Therefore, there exists $m_{H} \in M_{H}$ that vetoes $Y \cup\{x\}$. Since $x^{\prime}$ is neither vetoed by $m_{J}$ nor by $m_{H}, g\left(m_{J}, m_{H}\right)=\left\{x^{\prime}\right\}$. Consider a unanimous preference profile $>=\left(>_{1}, \ldots,>_{n}\right)$ such that $x>_{i} x^{\prime}>_{i} y$ for any $y \neq x, x^{\prime}$ and any $i=1, \ldots, n$. Note that $x^{\prime}$ is Pareto dominated by $x$ but at $\left(m_{J}, m_{H}\right)$, both coalitions veto $x$. No unilateral deviation is hence possible since no deviation can add $x$ to the support of the lottery outcome. Hence, SD implies that $\left(m_{J}, m_{H}\right)$ is a Nash equilibrium that selects a Pareto dominated alternative, a contradiction.

The proof of the lemma can be derived by applying the same logic to a finite sequence of sets $X=X_{1}, \ldots, X_{s}=X^{\prime}$ with $\#\left(X_{i} \cap X_{i+1}\right)=\# X-1$ for each $i \in\{1, \ldots, s-1\}$.

Lemmas 2 and 3 jointly prove that, for each $f$ Pareto efficient and implementable, the implementing mechanism $g$ is neutral-on-its-vetoes. That is, for each mechanism $g$ and each coalition $J$, we can define the number $v_{g}(J)$ that stands for the maximal number of alternatives that coalition $J$ can veto.

For each coalition $J$, we denote by $U\left(a,>_{J}\right)=\left\{x \in A \mid x>_{j} a \forall j \in J\right\}$ the upper contour set of the alternative $a$ at preference profile $>_{J}$. Thus, the upper contour set of $a$ for coalition $J$ includes all the alternatives which each member of $J$ prefers to $a$, which implies that we can express it as follows:

$$
U\left(a,>_{J}\right)=\left\{A \backslash\left(\{a\} \cup L\left(a,>_{J}\right)\right\}\right.
$$

The next lemma shows that for any partition of the voters, the sum of their respective veto powers adds up to $k-1$

Lemma 4. Assume the preference domain $\kappa(>)$ satisfies completeness, SD and PREX. If the mechanism g coalitionally implements a Pareto-efficient SCR $f$, then for any partition $(J, H)$ of $I$, $v_{g}(J)+v_{g}(H)=k-1$.

Proof. Consider a partition $(J, H)$ of $I$. Consider the following preference profile $>$ : 
1. $\forall j, j^{\prime} \in J,>_{j}=>_{j^{\prime}}$ and $\forall h, h^{\prime} \in H,>_{h}=>_{h^{\prime}}$

2. $\forall j \in J$ and $h \in H, p_{r}\left(>_{j}\right)=p_{k+1-r}\left(>_{h}\right)$, so the preference of $J$ and $H$ are opposed.

3. $\forall j, j^{\prime} \in J, \geq_{j}^{*}=z_{j^{\prime}}^{*}$ and $\forall h, h^{\prime} \in H, \geq_{h}^{*}=\geq_{h^{\prime}}^{*}$

Observe that for each $x \in X, U\left(x,>_{J}\right) \cap U\left(x,>_{J}\right)=\emptyset$ with $\left|U\left(x,>_{J}\right) \cup U\left(x,>_{H}\right)\right|=k-1$.

Consider some equilibrium $m$ such that $g(m)=\{a\}$ for some $a \in A$. Assume that for each voter $i \in I \geq_{i}^{*}$ satisfies PREX for $a$.

Since the preferences of all members in each coalition coincide, then $U\left(a,>_{J}\right)$ is vetoed by coalition $H$. To see why, assume by contradiction that coalition $H$ does not veto $U\left(a,>_{J}\right)$. Then, there is some $m_{J}^{\prime}$ such that $\operatorname{suppg}\left(m_{J}^{\prime}, m_{-J}\right) \cap U\left(a,>_{J}\right) \neq \emptyset$. Yet, since $\forall j, j^{\prime} \in J, \geq_{j}^{*}=\geq_{j^{\prime}}^{*}$, any deviation by coalition $J$ is self-enforcing, proving that $m$ is not coalition-proof. Similarly, we can prove that $U\left(a,>_{H}\right)$ is vetoed by coalition $J$. Thus, $v_{g}(H) \geq\left|U\left(a,>_{J}\right)\right|$ and $v_{g}(J) \geq \mid U\left(a,>_{H}\right.$ ) $\mid$. By construction of $>,\left|U\left(a,>_{J}\right)\right|+\left|U\left(a,>_{H}\right)\right|=k-1$, so $v_{g}(J)+v_{g}(H) \geq k-1$. In order for mechanism $g$ to be well-defined, $v_{g}(J)+v_{g}(H) \leq k-1$ (at least some alternative should be left non-vetoed). Thus, $v_{g}(J)+v_{g}(H)=k-1$.

\section{Proof of Theorem 2.}

Lemmas 2-4 have proved that $g$ is neutral-on-its-vetoes, that the veto power of different coalitions is additive and that for each partition $(J, K)$ of the voters their respective veto power $v_{g}(J)$ and $v_{g}(K)$ adds up to $k-1$.

As a final claim, we show that for any preference profile $>$, an equilibrium outcome is not among the $v_{g}(i)$ worse alternatives for any player $i$. To prove the claim, let $m \in N^{g}\left(\geq^{*}\right)$ be an equilibrium and $x$ its outcome. Assume that $x$ is among the v1 worst alternatives of player 1 . As previously argued, player 1 has a veto power of $v_{g}(1)$. Let $m_{i}^{\prime}$ denote the strategy of player $i$ that vetoes his worst $v_{i}$ alternatives. For any strategy $m_{-i} \in M_{-i}$, the support of $g\left(m_{i}^{\prime}, m_{-i}\right)$ is included in player $i$ 's top $k-v_{g}(i)$ alternatives. However, due to PREX, player $i$ prefers any such lottery to $x$, which proves that player $i$ has a profitable deviation and thus $m$ is not an equilibrium, which concludes the proof of the theorem.

\section{A.2 Proof of Theorem 4}

Let $f$ be a Pareto-efficient SCR coalitionally implementable by some deterministic mechanism $g$ satisfying NGC. We now show that $f$ is dictatorial. Note that Lemmas 2, 34 and 5 apply also for deterministic mechanisms. Indeed, by focusing on deterministic mecha- 
nisms, $g(M) \subseteq A$ which implies the voters' preferences over $g(M)$ directly satisfy completeness. Morever, the deterministic versions of SD and PREX are also satisfied since again the mechanism is deterministic.

Then the following two observations remain true:

- one voter, say voter $i$, is a dictator, so $v(i)=k-1$;

- or there are at least 2 voters with veto power of at least 1.

Indeed, if voter $i$ has strictly less than $k-1$ vetoes there is some coalition which does not include voter $i$ which has at least 1 veto. Then by Lemma 5 , at least one voter in this coalition has a veto power of at least 1.

Let $J \in C^{I}$ be the set of voters with strictly positive number of vetoes. Lemma 3 implies that $g$ is neutral-on-its-vetoes so that any coalition $K \in C^{I}$ can veto any set of alternatives with cardinality at most $v_{g}(K)$. Consider the following strategy profile $m$ :

1. $a_{1} \notin g\left(M_{-j}, m_{j}\right)$ for all $j \in J$ for some $a_{1} \in A$;

2. $g(m)=x$ for some $x \in A$.

Point 1 is possible since set $J$ contains at least 2 voters. Thus, if they both veto $a_{1}$ at the same time, this alternative is not in the attainable set of any of them. Point 2. is possible since $g$ is deterministic, thus the outcome is a singletone for any profile $m$.

Take now the following preference profile: for each $i \in I, a_{1}>_{i} x>_{i} y$ for any $y \neq a_{1}, x$. Then for every voter $j$, for any $m_{j}^{\prime} \in M_{j}, a_{1} \notin g\left(m_{j}^{\prime}, m_{-i}\right)$. That means that after any deviation of any voter $j$, alternative $a_{1}$ is still vetoed by other voters. Then, the best outcome any voter can achieve by deviation is $x$. Since $g(m)=x, m$ is a Nash equilibrium that implements $x$, an alternative Pareto-dominated by $a_{1}$. This contradicts $f$ being Pareto-efficient.

\section{A.3 Proof of Proposition 5}

In order to prove the first part of the proposition, the next lemma proves that if $g$ is the implementing mechanism in Theorem 2 and satisfies NGC then $v_{g}(J)$ is additive, so that for each pair of coalitions $J_{1}, J_{2}$ with $J_{1} \cap J_{2}=\emptyset$ and $J=J_{1} \cup J_{2}, v_{g}\left(J_{1} \cup J_{2}\right)=v_{g}\left(J_{1}\right)+v_{g}\left(J_{2}\right)$. Finally, Lemma 6 proves that any coalitionally implementable $f$ selects all the alternatives prescribed by some $V R P_{v}$ rule. 
Lemma 5. Assume that the preference domain $\kappa(>)$ satisfies completeness, SD and PREX. If the mechanism $g$ coalitionally implements a Pareto-efficient SCR $f$ and satisfies NGC, then $v_{g}(\cdot)$ is additive.

Proof. Consider any two disjoint coalitions $J_{1}$ and $J_{2}$ such that $J_{1} \cup J_{2}=J$. Consider some $X \subset A$ such that $X \in \operatorname{veto}(g, J)$ and $|X|=v_{g}(J)$. According to NGC, there are $Y, Z \subset A$ such that $Y \in \operatorname{veto}\left(g, J_{1}\right), Z \in \operatorname{veto}\left(g, J_{2}\right)$ and $Y \cup Z=X$. Then there are 2 possibilities:

1. either $v_{g}\left(J_{1}\right)=|Y|$ and $v_{g}\left(J_{2}\right)=|Z|$

2. or $v_{g}\left(J_{1}\right)>|Y|\left(\right.$ or $\left.v_{g}\left(J_{2}\right)>|Z|\right)$

If $v_{g}\left(J_{1}\right)>|Y|$ or $v_{g}\left(J_{2}\right)>|Z|$ then $v_{g}\left(J_{1}\right)+v_{g}\left(J_{2}\right)>v_{g}(J)$, since the mechanism $g$ is neutralon-its-vetoes by Lemma 3. Thus, it means that the joint coalition has veto rights smaller than the sum of veto rights of its 2 parts. However, this cannot be the case. To see this take some profile $m$ such that $m_{J_{1}}$ and $m_{J_{2}}$ are vetoing two disjoint sets of sizes $v_{g}\left(J_{1}\right)$ and $v_{g}\left(J_{2}\right)$ respectively. Such profile exists since $g$ is neutral-on-its-vetoes and $v_{g}\left(J_{1}\right)+v_{g}\left(J_{2}\right) \leq k-1$ since $g$ is well-defined. Since the set of alternatives vetoed by $J_{1} \cup J_{2}$ should at least include the alternatives vetoed by $J_{1}$ and $J_{2}, v_{g}(J) \geq v_{g}\left(J_{1}\right)+v_{g}\left(J_{2}\right)$. Then the only possibility is that $v_{g}\left(J_{1}\right)=|Y|$ and $v_{g}\left(J_{2}\right)=|Z|$. Thus $v_{g}(J)=v_{g}\left(J_{1} \cup J_{2}\right)=v_{g}\left(J_{1}\right)+v_{g}\left(J_{2}\right)$, as required.

Lemma 6. Assume that domain $\kappa(>)$ satisfies SD and PREX. If a Pareto-efficient SCR $f$ is coalitionally implementable by some DE mechanism satisfying NGC then $f \supseteq V R P_{v}$ fo some $v$.

Proof. Consider some DE mechanism $g$ satisfying NGC and coalitionally implementing $f$.

By Theorem 2, the veto function of mechanism $g$ is neutral-on-its-vetoes, additive and for each partition $J, K$ of $I, v_{g}(J)+v_{g}(K)=k-1$. Thus, there exists some veto vector $v=\left(v_{1}, \ldots, v_{n}\right)$ such that $v_{g}(i)=v_{i}$ and $v_{g}(J)=\sum_{j \in J} v_{j}$.

Consider some preference profile $>$. Take some alternative $a$ such that $\operatorname{SV}_{v}(m)=\{a\}$ for some $m \in N^{\mathrm{SV}_{v}}\left(\geq^{*}\right)$.

Now consider a strategy $\widetilde{m}$ for the mechanism $g$ such that each voter $i$ vetoes under $\widetilde{m}$ the alternatives in $m_{i}$. In other words, we construct a profile for mechanism $g$ such that each voters veto the same alternatives as in an equilibrium profile for the mechanism $\mathrm{SV}_{v}$. Such mechanism indeed exists since $g$ is neutral-on-its-vetoes and $v_{g}(i)=v_{i}$ for each voter $i \in I$. Then $g(\widetilde{m})=\{a\}$ since all other alternatives are vetoed.

Now consider any coalition $J \in C^{I}$. If a profitable deviation from coalition $J$ from $\widetilde{m}$ under a mechanism $g$ exists, say $\widetilde{m}_{J}^{\prime}$ then $\operatorname{supp}\left(g\left(\widetilde{m}_{J}^{\prime}, m_{-J}\right)\right) \cap U\left(a,>_{J}\right) \neq \emptyset$. However, by construction 
of the profile $\widetilde{m}$ all alternatives in $U\left(a,>_{J}\right)$ are vetoed by voters in $I \backslash J$. Thus, $\widetilde{m}$ is a Nash equilibrium.

But then we have for every $>\in R$ :

$$
f(>)=\bigcup_{\widetilde{m} \in N^{g}\left(\geq^{*}\right)} g(\widetilde{m}) \supseteq \bigcup_{m \in N^{S V_{v}}\left(\geq^{*}\right)} \operatorname{SV}_{v}(m)=\operatorname{VRP}_{v}(>)
$$

The equalities follow respectively from the fact that $g$ coalitionally implements $f$ and $\mathrm{SV}_{v}$ coalitionally implements $\mathrm{VRP}_{v}$.

The second part of Proposition 5 shows that when $k \geq n$, at least some voter has positive veto power. We start with the simple case of 3 voters and 3 alternatives and show that at least 1 voter should have a veto power of 1 .

1. $n=k=3$.

Consider the preference profile in Table 1 that describes a Condorcet cycle:

\begin{tabular}{c|c|c}
$>_{1}$ & $>_{2}$ & $>_{3}$ \\
\hline$a$ & $b$ & $c$ \\
$b$ & $c$ & $a$ \\
$c$ & $a$ & $b$
\end{tabular}

Table 1

Assume that $\geq^{*}$ satisfies PREX and fix some DE implementing mechanism $g$. Assume that $a$ is an equilibrium outcome. Thus, there exists some $m \in N^{g}\left(\geq^{*}\right)$ with $g(m)=\{a\}$.

Notice that since $a$ is the worst alternative for voter 2 it must be that $\{b, c\} \in \operatorname{veto}(g, 1 \cup 3)$. Additionally, due to PREX it must be that $c$ is blocked by coalition of voters 1 and 2, so $\{c\} \in \operatorname{veto}(g, 1 \cup 2)$. There are 2 possibilities:

1. $c$ is vetoed by voter 1 under $m$. Thus $\{c\} \in \operatorname{veto}(g, 1)$.

2. $c$ is not vetoed by voter 1 under $m$, but it is vetoed by coalitions $1 \cup 3$ and $2 \cup 3$. Then consider the preference profile in Table 2, in which 2 and 3 share identical preferences.

\begin{tabular}{c|c|c}
$>_{1}^{\prime}$ & $>_{2}^{\prime}$ & $>_{3}^{\prime}$ \\
\hline$a$ & $c$ & $c$ \\
$b$ & $a$ & $a$ \\
$c$ & $b$ & $b$
\end{tabular}

Table 2 
Assume that $\geq_{2}^{\prime *}$ and $\geq_{3}^{\prime *}$ are identical and satisfy PREX. We claim that $m$ is a Nash equilibrium in the profile $>^{\prime}$. Voter 1 has no profitable deviation since $a$ is her most preferred alternative. Voter 2 gets her best among nonvetoed by voters 1 and 3 , since $c$ is vetoed by coalition of voters 1 and 3. The same applies to voter 3. However, $m$ is not coalition-proof. Indeed, since $c$ is not vetoed by voter 1 , the coalition $2 \cup 3$ has a deviation $\left(m_{2}^{\prime}, m_{3}^{\prime}\right)$ such that $c \in \operatorname{supp}\left(g\left(m_{1}, m_{2}^{\prime}, m_{3}^{\prime}\right)\right.$ and according to PREX this deviation is profitable. Since the preference profiles of voters 2 and 3 are identical this deviation is also self-enforcing. Thus, it is not possible that in the profile $>$ under equilibrium $m c$ is vetoed by coalitions $1 \cup 2$ and $1 \cup 3$ but not by voter 1 individually.

Then, $\{c\} \in \operatorname{veto}(g, 1)$. Since, according to Theorem 2 , the mechanism $g$ is neutral-on-itsvetoes it follows that $v_{g}(1) \geq 1$.

Notice that if $b$ or $c$ are equilibrium outcomes in Table 1 , a symmetric logic applies and leads to $v_{g}(2) \geq 1$ and $v_{g}(3) \geq 1$ correspondingly, concluding the proof of $n=k=3$.

In the next step we consider 3 voters and 4 alternatives and show that the sum of individual veto powers should be at least 2 .

2. $n=3$ and $k=4$

Consider some Pareto-efficient mechanism $g$ such that $N^{g}\left(\geq^{*}\right)=C^{g}\left(\geq^{*}\right)$ for all $>, \geq^{*} \in \kappa(>)$. Consider the preference profile $>$ of Table 3 .

\begin{tabular}{c|c|c}
$>_{1}$ & $>_{2}$ & $>_{3}$ \\
\hline$a$ & $b$ & $c$ \\
$b$ & $c$ & $a$ \\
$c$ & $a$ & $b$ \\
$d$ & $d$ & $d$
\end{tabular}

Table 3

Since the mechanism $g$ is Pareto-efficient, only $a, b$ or $c$ can be equilibrium outcomes.

W.l.o.g. assume that $a$ is an equilibrium outcome. Then, by the same logic as in the case with 3 alternatives $v_{g}(1) \geq 1$.

Now consider the preference profile $>^{\prime}$ depicted by Table 4 and assume that $\geq^{\prime *}$ satisfies PREX.

Note that in this profile $d$ can not be an outcome since $v_{g}(1) \geq 1$. Then there are 2 possibilities:

1. There is some $m \in N^{g}\left(\geq^{\prime *}\right)$ such that $g(m)=\{a\}$. Then $\{d, c\}$ should be vetoed by coali- 


\begin{tabular}{c|c|c}
$>_{1}^{\prime}$ & $>_{2}^{\prime}$ & $>_{3}^{\prime}$ \\
\hline$a$ & $d$ & $d$ \\
$b$ & $b$ & $c$ \\
$c$ & $c$ & $a$ \\
$d$ & $a$ & $b$
\end{tabular}

Table 4

tion $1 \cup 2,\{d, b, c\}$ should be vetoed by coalition $1 \cup 3$. If we assume that $d$ is not vetoed by voter 1 individually, but only by 2 coalitions then we arrive to the same contradiction as in the 3 alternatives case. Similarly, $c$ must be vetoed by voter 1 individually. Thus $v_{g}(1) \geq 2$.

2. $a$ is never an equilibrium outcome. Then, w.l.o.g assume that there is some $m^{\prime} \in N^{g}\left(\geq^{\prime *}\right)$ such that $g\left(m^{\prime}\right)=\{b\}$. Then $a$ must be vetoed by coalition $2 \cup 3, d$ must be vetoed by coalition $(1,3)$ and $d, c, a$ must be vetoed by coalition $1 \cup 2$. If $a$ is not vetoed individually by voter 2 then we can use the same logic as before to show that there exists a profile in which $m^{\prime}$ is a Nash equilibrium, but not coalition-proof. Thus, a must be vetoed by voter 2 individually. Thus, $v_{g}(2) \geq 1$. Combining this with $v_{g}(1) \geq 1$ we get that $\sum_{i \in I} v_{g}(i) \geq 2$.

Now we can generalize that for the arbitrary settings with $k \geq n$.

\section{3. $k \geq n$.}

The first preference profile to consider is included in Table 5. In this profile, denoted $>$, the first $n$ alternatives form a Condorcet cycle whereas all the remaining alternatives are Pareto-dominated.

\begin{tabular}{c|c|c|c|c}
$>_{1}$ & $>_{2}$ & $>_{3}$ & $\ldots$ & $>_{n}$ \\
\hline$a_{1}$ & $a_{2}$ & $a_{3}$ & $\ldots$ & $a_{n}$ \\
$a_{2}$ & $a_{3}$ & $a_{4}$ & $\ldots$ & $a_{1}$ \\
$a_{3}$ & $a_{4}$ & $a_{5}$ & $\ldots$ & $a_{2}$ \\
$\vdots$ & $\vdots$ & $\vdots$ & $\vdots$ & $\vdots$ \\
$a_{n}$ & $a_{1}$ & $a_{2}$ & $\ldots$ & $a_{n-1}$ \\
$a_{n+1}$ & $a_{n+1}$ & $a_{n+1}$ & $\ldots$ & $a_{n+1}$ \\
$\vdots$ & $\vdots$ & $\vdots$ & $\ldots$ & $\vdots$ \\
$a_{k}$ & $a_{k}$ & $a_{k}$ & $\ldots$ & $a_{k}$
\end{tabular}

Table 5

We assume that $\geq^{*}$ satisfies PREX. W.l.o.g, assume that $a_{1}$ is an equilibrium outcome of $g$ at $>$. Then alternative $a_{n}$ must be vetoed by all coalitions of size $n-1$ which include voter 1. Then, as in the case with 3 voters, there are 2 cases: 
1. $a_{n}$ is vetoed by voter 1 . Them $v_{g}\left(a_{n}\right) \geq 1$.

2. $a_{n}$ is vetoed by all coalitions of size $n-1$ which include voter 1 , but not by voter 1 individually. In this case consider the preference profile $>^{\prime}$ in Table 6.

\begin{tabular}{c|c|c|c|c}
$>_{1}$ & $>_{2}$ & $>_{3}$ & $\ldots$ & $>_{n}$ \\
\hline$a_{1}$ & $a_{n}$ & $a_{n}$ & $\ldots$ & $a_{n}$ \\
$a_{n}$ & $a_{1}$ & $a_{1}$ & $\ldots$ & $a_{1}$ \\
$a_{2}$ & $a_{2}$ & $a_{2}$ & $\ldots$ & $a_{2}$ \\
$\vdots$ & $\vdots$ & $\vdots$ & $\vdots$ & $\vdots$ \\
$a_{k}$ & $a_{k}$ & $a_{k}$ & $\ldots$ & $a_{k}$
\end{tabular}

Table 6

Assume that $\geq^{\prime *}$ satisfies PREX. Assume also that for voters $2,3, \ldots, n$ preference extension is identical.

In this case, $m$ is a Nash equilibrium. However, since $\geq^{*}$ satisfies PREX, the preferences of voters $2,3, \ldots n$ are identical and $a_{n}$ is not vetoed by voter 1 , coalition $2 \cup 3 \cup \ldots \cup n$ has a profitable self-enforcing deviation. Thus, $m$ is not coalition-proof.

Then we can conclude that $v_{g}(1) \geq 1$.

In the next step we consider the preference profile $>$ " included in Table 7. Assume that $\geq{ }^{\prime \prime}$ satisfies PREX.

\begin{tabular}{c|c|c|c|c}
$>_{1}$ & $>_{2}$ & $>_{3}$ & $\ldots$ & $>_{n}$ \\
\hline$a_{1}$ & $a_{n+1}$ & $a_{n+1}$ & $\ldots$ & $a_{n+1}$ \\
$a_{2}$ & $a_{2}$ & $a_{3}$ & $\ldots$ & $a_{n}$ \\
$a_{3}$ & $a_{3}$ & $a_{4}$ & $\ldots$ & $a_{1}$ \\
$a_{4}$ & $a_{4}$ & $a_{5}$ & $\ldots$ & $a_{2}$ \\
$\vdots$ & $\vdots$ & $\vdots$ & $\vdots$ & $\vdots$ \\
$a_{n}$ & $a_{n}$ & $a_{1}$ & $\ldots$ & $a_{n-2}$ \\
$a_{n+2}$ & $a_{1}$ & $a_{2}$ & $\ldots$ & $a_{n-1}$ \\
$a_{n+3}$ & $a_{n+2}$ & $a_{n+2}$ & $\ldots$ & $a_{n+2}$ \\
$\vdots$ & $\vdots$ & $\vdots$ & $\ldots$ & $\vdots$ \\
$a_{n+1}$ & $a_{k}$ & $a_{k}$ & $\ldots$ & $a_{k}$
\end{tabular}

Table 7

In this profile alternative $a_{1}, \ldots, a_{n}$ form a Condorcet cycle and the alternatives $a_{n+2}, \ldots, a_{k}$ are Pareto-dominated. However, alternative $a_{n+1}$ is the worst for voter 1 while it is the best for all other voters.

Note that since $v_{g}(1) \geq 1, a_{n+1}$ cannot be an equilibrium outcome. Assume that there is 
some profile $m^{\prime \prime} \in N^{g}\left(>^{\prime \prime}\right)$ such that $g\left(m^{\prime \prime}\right)=\left\{a_{1}\right\}$. Then $a_{n}$ and $a_{n+1}$ must be vetoed by each coalition of size $n-1$ which includes voter 1 since these alternatives are preferred to $a_{1}$ by all voters except voter 1 . Thus, applying the same logic as before we can conclude that $v_{g}(1) \geq 2$.

If there is no such $m^{\prime \prime}$ consider w.l.o.g. some $m^{\prime \prime \prime} \in N^{g}\left(>^{\prime \prime}\right)$ with $g\left(m^{\prime \prime \prime}\right)=\left\{a_{2}\right\}$. Then $a_{n+1}$ must be vetoed by all coalitions of size $n-1$ which include voter 1 and $a_{1}$ must be vetoed by all coalitions of size $n-1$ which include voter 2. Applying the same logic as before that leads to $v_{g}(1) \geq 1$ and $v_{g}(2) \geq 1$.

According to the results of this step we move to the next profile $>^{\prime \prime \prime}$. If at this stage $v_{g}(1) \geq 2$ then $>^{\prime \prime \prime}$ is as indicated in Table 8:

\begin{tabular}{c|c|c|c|c}
$>_{1}^{\prime \prime \prime}$ & $>_{2}^{\prime \prime \prime}$ & $>_{3}^{\prime \prime \prime}$ & $\ldots$ & $>_{n}^{\prime \prime \prime}$ \\
\hline$a_{1}$ & $a_{n+1}$ & $a_{n+1}$ & $\ldots$ & $a_{n+1}$ \\
$a_{2}$ & $a_{n+2}$ & $a_{n+2}$ & $\ldots$ & $a_{n+2}$ \\
$a_{3}$ & $a_{2}$ & $a_{3}$ & $\ldots$ & $a_{n}$ \\
$a_{4}$ & $a_{3}$ & $a_{4}$ & $\ldots$ & $a_{1}$ \\
$\vdots$ & $\vdots$ & $\vdots$ & $\vdots$ & $\vdots$ \\
$a_{n}$ & $a_{n-1}$ & $a_{n}$ & $\ldots$ & $a_{n-3}$ \\
$a_{n+3}$ & $a_{n}$ & $a_{1}$ & $\ldots$ & $a_{n-2}$ \\
$a_{n+4}$ & $a_{1}$ & $a_{2}$ & $\ldots$ & $a_{n-1}$ \\
$a_{n+5}$ & $a_{n+3}$ & $a_{n+3}$ & $\ldots$ & $a_{n+3}$ \\
$\vdots$ & $\vdots$ & $\vdots$ & $\ldots$ & $\vdots$ \\
$a_{n+1}$ & $a_{k-1}$ & $a_{k-1}$ & $\ldots$ & $a_{k-1}$ \\
$a_{n+2}$ & $a_{k}$ & $a_{k}$ & $\ldots$ & $a_{k}$
\end{tabular}

Table 8

Since $v_{g}(1) \geq 2$, alternatives $a_{n+1}$ and $a_{n+2}$ are not equilibrium outcomes. Thus only the alternatives that form the Condorcet cycle can be equilibrium outcomes. Then, by the same logic as before one voter has the additional veto power.

If at the previous step, $v_{g}(1) \geq 1$ and $v_{g}(2) \geq 1$ then consider the profile $>^{\prime \prime \prime}$ in Table 9.

Then again only the alternatives in the Condorcet cycle can be equilibrium outcomes, and so we get an additional veto for some voter.

After repeating this procedure we finally arrive to the situation in which the sum of vetoes of individual voters is $k-n$ and we can not construct a profile with Pareto-dominated alternatives anymore. Indeed in the last stage, the alternatives $a_{1}, \ldots, a_{n}$ form a Condorcet cycle, for each voter $i$ there are $v_{g}(i)$ alternatives preferred less than any alternative in the Condorcet cycle, other alternatives each voter $i$ prefers to each alternative in the Condorcet cycle. By the usual logic, if one alternative in the Condorcet cycle is elected then one voter 


\begin{tabular}{c|c|c|c|c}
$>_{1}^{\prime \prime \prime}$ & $>_{2}^{\prime \prime \prime}$ & $>_{3}^{\prime \prime \prime}$ & $\ldots$ & $>_{n}^{\prime \prime \prime}$ \\
\hline$a_{n+2}$ & $a_{n+1}$ & $a_{n+1}$ & $\ldots$ & $a_{n+1}$ \\
$a_{1}$ & $a_{2}$ & $a_{n+2}$ & $\ldots$ & $a_{n+2}$ \\
$a_{2}$ & $a_{3}$ & $a_{3}$ & $\ldots$ & $a_{n}$ \\
$a_{3}$ & $a_{4}$ & $a_{4}$ & $\ldots$ & $a_{1}$ \\
$\vdots$ & $\vdots$ & $\vdots$ & $\vdots$ & $\vdots$ \\
$a_{n}$ & $a_{1}$ & $a_{2}$ & $\ldots$ & $a_{n-1}$ \\
$a_{n+2}$ & $a_{n+1}$ & $a_{1}$ & $\ldots$ & $a_{n+1}$ \\
$a_{n+3}$ & $a_{n+3}$ & $a_{n+2}$ & $\ldots$ & $a_{n+2}$ \\
$\vdots$ & $\vdots$ & $\vdots$ & $\ldots$ & $\vdots$ \\
$a_{k}$ & $a_{k}$ & $a_{k-1}$ & $\ldots$ & $a_{k-1}$ \\
$a_{n+1}$ & $a_{n+2}$ & $a_{k}$ & $\ldots$ & $a_{k}$
\end{tabular}

Table 9

has additional veto which leads to $(k-n+1)$ 'st veto assigned, as desired.

\section{B Proof of Proposition 6}

The proof of Proposition 6 is located at the end of this section; prior to it, we state several claims that will be useful in the proof.

In Claims 1 and 2 we assume that the set $N^{g}\left(\geq^{*}\right)$ is nonempty for each preference profile $>$ and each $\geq^{*} \in \kappa(>)$.

Claim 1: Fix some $m \in N^{g}\left(\geq^{*}\right)$. If there is $J \subset I$ with $|J|=2$ such that for $\{a, b\} \in A \backslash v^{1}, a>_{j} b$ for each $j \in J$ and $b>_{I \backslash J} a$, then $a \in c^{j}$ for all $j \in J$ and $v^{1} \in c^{j}$ for all $j \in J \cap\{2,3\}$. Moreover $g(m)=\{a\}$.

According to Claim 1, if we fix some equilibrium and some vetoed alternative $v^{1}$, whenever there are exactly 2 voters which have the same preference over the remaining alternatives, then their preferred alternative, say $a$, gets elected. In equilibrium, they both announce $a$ and $v^{1}$.

Proof of Claim 1: Consider some equilibrium profile $m$. Assume first that voters 2 and 3 have the same preferences over $A \backslash v^{1}$. Without loss of generality assume that $A \backslash v^{1}=\{a, b\}$, $v^{1}=\{c\}, a>_{2} b$ and $a>_{3} b$. By contradiction, assume that one of them, say voter 3 , announces something different from $(a, c)$, so $(a, b)$ or $(b, c)$.

If voter 3 is nominated, so either $n^{1}=\{3\}$ or $n^{2}=\{3\}$ or both, then she has a profitable deviation to announce $(a, c)$. Indeed, it maximizes the probability of $a$ in the resulting lottery 
with respect to $b$ (alternative $c$ is vetoed).

If voter 3 is not nominated, then, voter 1 nominates voter 2. By the same logic as for voter 3 , voter 2 , when nominated, announces $(a, c)$, so $c^{2}=(a, c)$. In this case, since $b>_{1} a$, voter 1 has a profitable deviation to set $n^{1}=\{3\}$ and by this to increase the probability of $b$ in the resulting lottery.

If voters 1 and 2 or voters 1 and 3 have the same preference over $A \backslash v^{1}$ the same logic applies.

Now we show that $a$ is indeed the outcome. Assume $b \in C(m) \backslash v^{1}$, so $b$ is in the support of the resulting lottery. Also let $J$ be the set of voters who prefer $a$ to $b$, so $|J|=2$. Since, according to the previous discussion, no voter who prefers $a$ to $b$ announces $b$ it must be the case that the voter who prefers $b$ to $a$ is nominated by one of the voters who prefer $a$ to $b$, say voter $j$. Formally, if $b \in \operatorname{supp}(g(m))$ then $n^{j}=I \backslash J$ for some $j \in J$. But then voter $j$ has a profitable deviation to nominate another voter who prefers $a$ to $b$, so to set $n^{j}=J \backslash\{j\}$.

Claim 2: In any $m \in N^{g}\left(\geq^{*}\right)$, if $A \backslash v^{1}=\{a, b\}$ and $a>_{i} b$ for each $i \in I$ then $g(m)=\{a\}$.

Proof of Claim 2: By contradiction, assume that there is some $b \neq a$ such that $b \in \operatorname{supp}(g(m))$. In this case, there is some voter $i \in I$ such that $n^{j}=\{i\}$ for some $j \in I, j \neq i$ and $b \in c^{i}$. Then, she has a profitable deviation, which implies that $m$ is not an equilibrium.

Corollary 1: In any Nash equilibrium, the alternative in $A \backslash v^{1}$ preferred by the majority of voters is the outcome.

Corollary 2: Any Nash equilibrium is DE.

Claim 3: The mechanism $g$ always admits an equilibrium.

Proof of Claim 3: Denote by $\varphi\left(>, v^{1}\right)$ the alternative in $A \backslash v^{1}$ preferred by at least 2 voters.

Take an arbitrary preference profile $>$ and consider the following strategy profile $m . v^{1}$ is the least preferred alternative for voter 1 in the set $\left\{x \in A \mid x=\arg \max _{y \in A} \varphi(>, y)\right\}$. Thus, voter 1 vetoes her least preferred alternative among those which lead to the outcome $\varphi\left(>, v^{1}\right)$. Note that in this case voter 1 always vetoes an alternative which she prefers less than $\varphi\left(>, v^{1}\right)$.

Then 2 voters have identical preferences over the remaining 2 alternatives with $\varphi\left(>, v^{1}\right)$ being the most preferred. Let then $c^{1}=\varphi\left(>, m_{1}^{1}\right), c_{1}^{2}=c_{1}^{3}=v^{1}, c_{2}^{2}=c_{3}^{3}=\varphi\left(>, v^{1}\right)$. Let also 2 voters who have the same preference among non-vetoed alternatives to nominate each other. Such profile $m$ is a Nash equilibrium. Indeed, voter 1 either gets her best outcome, or she is not nominated, and she vetoes the worst alternative among announced by nominated voters. Voters 2 and 3 either get their best among non vetoed or are not nominated, thus, do not 
affect the outcome.

Claim 4: Any Nash equilibrium of $g$ is Pareto-efficient.

Proof of Claim 4: By Corollary 1 a Nash equilibrium outcome can be Pareto-inefficient only if a Pareto-dominant alternative is vetoed by voter 1. Assume that such alternative is $a$ and $v^{1}=\{a\}$. Since the equilibrium is deterministic there is at least some nominated voter among 2 and 3 who announces $a$. Indeed, otherwise there would be a nominated voter who announces 2 remaining alternatives which would lead to a lottery. Then voter 1 has a profitable deviation - to veto her worst alternative among announced by voters 2 and 3 and to set $c^{1}=\{a\}$. Thus, a Pareto-inefficient alternative can not be an equilibrium outcome.

Claim 5: Any Nash equilibrium of $g$ is coalition-proof.

Proof of Claim 5: Consider some $m \in N^{g}\left(\geq^{*}\right)$ of $g$ such that $g(m)=\{a\}$ and assume that there exists coalition $J$ and a self-enforcing deviation $m_{J}^{\prime}$ such that $g\left(m_{J}^{\prime}, m_{-J}\right) \geq_{j}^{*} g(m)$ for all $j \in J$. Notice that since mechanism $g$ is Pareto-efficient and $m$ is a Nash equilibrium, $|J|=2$. That means that for some coalition of 2 voters $J$ there is a non-vetoed alternative preferred to $a$ by both voters since $m$ is deterministic. There are 2 possibilities.

1. Coalition $2 \cup 3$ has a deviation. Thus, there exists an alternative $b$ such that $v^{1} \neq\{b\}$ and such that $b>_{2} a$ and $b>_{3} a$. By the construction of the mechanism $g$ at least one of the voters 2 or 3 is nominated. Without loss of generality, assume it is voter 2 . If $b$ is not in the outcome, that means that $b \notin c^{2}$. Thus, $a \in c^{2}$ since there are 3 alternatives. Then voter 2 has a profitable deviation to announce $b$ instead of $a$. This will lead to the resulting lottery being a lottery between $b$ and $a$. Thus it is a profitable deviation for this voter which contradicts $m$ being a Nash equilibrium.

2. Coalition $1 \cup 2$ or $1 \cup 3$ has a deviation. Without loss of generality assume that it is coalition 1,2. Thus there is some alternative $b$ such that $b>_{1} a$ and $b>_{2} a$. If $v^{1}=\{b\}$, since the outcome is deterministic $b \in c^{i}$ for some $i \in\{2,3\}$. Then voter 1 has a profitable deviation to veto her worst among announced by 2 and 3 and to set $n^{1}=\{i\}$. Then the outcome is either $b$, the lottery among $a$ and $b$, or the lottery among $b$ and another alternative voter 1 prefers to $a$. Thus, it is indeed a profitable deviation for voter 1 which contradicts $m$ being a Nash equilibrium.

If $v^{1} \neq b$ then the logic is the same as for coalition $2 \cup 3$.

Thus, no coalitional deviation is possible at any Nash equilibrium $g$.

Proof. We first show that $N^{g}\left(\geq^{*}\right) \subseteq f(>)$ Notice first, that since voter 1 has one veto in any 
equilibrium of $g$, the least preferred alternative of voter 1 is never elected.

Also because of Pareto-efficiency for any a such that $\{a\}=g(m)$ for some $m \in N^{g}\left(\geq^{*}\right)$, $l(a,>)=2$.

Now consider coalition $2 \cup 3$ and assume that some $a$ is elected such that $l\left(a,>_{2 \cup 3}\right)<1$. That means that $a$ is the least preferred for both voters. By Claims 1 and 2 the elected alternative is the one preferred by the majority in the set $A \backslash v^{1}$. Since voters 2 and 3 in this case have the same preference over non-vetoed alternatives, they are both part of the majority, thus their worst alternative cannot be the outcome. So $l\left(a,>_{2 \cup 3}\right) \geq 1$ for any $a$ selected in equilibrium.

Now consider the coalition $1 \cup 2$ and assume that some $a$ is elected with $l\left(a,>_{1 \cup 2}\right)<$ 2. Note that, in equilibrium, voter 1 always vetoes some alternative that she prefers less than the outcome, thus $l\left(a,>_{1 \cup 2}\right) \geq 1$. Among the remaining alternatives the one which is preferred by the majority is elected. Since one of the voters 1 or 2 is in this majority, her best among non-vetoed is elected in equilibrium. Thus, $l\left(a,>_{1 \cup 2}\right) \geq 2$.

The logic for coalition $1 \cup 3$ is identical.

To see that each alternative selected by $f$ is an equilibrium outcome of mechanism $g$ we construct an equilibrium profile of $g$ for any alternative $a \in f(>)$.

Take some $a \in f(>)$.

Case 1: $l\left(a,>_{1}\right)=1$. Assume that $b$ is the alternative such that $a>_{1} b$. Then set $v^{1}=\{b\}$. According to $f$ it must be that among 2 remaining alternatives $a$ and, say, $c$, voters 2 and 3 prefer $a$. Then set $c^{2}=(a, b), c^{3}=(a, b), c^{1}=\{a\}$ and $n^{2}=\{3\}, n^{3}=\{2\}$. Such profile is indeed a Nash equilibrium.

Case 2: $l\left(a,>_{1}\right)=2$. Thus, $a$ is the best alternative for voter 1 . Assume that $b, c$ are the remaining alternatives and $b>_{1} c$. It must be the case that there is some voter $i \in\{2,3\}$ such that $a>_{i} b$ or $a>_{i} c$. Indeed, otherwise $a \notin f(>)$. Without loss of generality assume that there is voter $i$ such that $a>_{i} b$. Then let $v_{1}=\{c\}, c^{1}=\{a\}, n^{1}=\{i\}, c^{i}=(a, c), n^{i}=\{1\}$. The strategy of the third voter does not matter in this case, such profile is a Nash equilibrium.

\section{References}

S. Barberà, F. Gul, and E. Stacchetti. Generalized median voter schemes and committees. Journal of Economic Theory, 61(2):262-289, 1993. 
J.-P. Benoît and E.A. Ok. Nash implementation without no-veto power. Games and Economic Behavior, 64(1):51-67, 2008.

B. D. Bernheim, B. Peleg, and M.D. Whinston. Coalition-proof Nash equilibria i. concepts. Journal of Economic Theory, 42(1):1-12, 1987.

F.J. Bierbrauer and M.F. Hellwig. Robustly coalition-proof incentive mechanisms for public good provision are voting mechanisms and vice versa. The Review of Economic Studies, 83 (4):1440-1464, 2016.

O. Bochet. Nash implementation with lottery mechanisms. Social Choice and Welfare, 28(1): 111-125, 2007.

O. Bochet and F. Maniquet. Virtual Nash implementation with admissible support. Journal of mathematical economics, 46(1):99-108, 2010.

A. Bogomolnaia, R. Holzman, and H. Moulin. Worst case in voting and bargaining. mimeo, Paris School of Economics, 2021.

K.C. Border and J.S. Jordan. Straightforward elections, unanimity and phantom voters. The Review of Economic Studies, 50(1):153-170, 1983.

T. Börgers and J. Li. Strategically simple mechanisms. Econometrica, 87(6):2003-2035, 2019.

L. Bouton, A. Llorente-Saguer, and F. Malherbe. Get rid of unanimity rule: the superiority of majority rules with veto power. Journal of Political Economy, 126(1):107-149, 2018.

F. Brandt. Set-monotonicity implies Kelly-strategyproofness. Social Choice and Welfare, 45 (4):793-804, 2015.

F. Brandt, V. Conitzer, U. Endriss, J. Lang, and A.D. Procaccia. Handbook of computational social choice. Cambridge University Press, 2016.

B. Caillaud and J. Tirole. Consensus building: How to persuade a group. American Economic Review, 97(5):1877-1900, 2007.

G.W. Cox and K.A. Shepsle. Majority cycling and agenda manipulation: Richard Mckelvey's contributions and legacy. A positive change in political science: The legacy of Richard McKelvey's most influential writings, 2007. 
G. De Clippel, K. Eliaz, and B. Knight. On the Selection of arbitrators. American Economic Review, 104:3434-58, 2014.

B. Dutta and A. Sen. A Necessary and Sufficient Condition for Two-person Nash Implementation. Review of Economic Studies, 58:121-128, 1991.

B. Dutta and A. Sen. Nash implementation with partially honest individuals. Games and Economic Behavior, 74:154-169, 2012.

A. Gershkov, B. Moldovanu, and X. Shi. Optimal voting rules. The Review of Economic Studies, 84(2):688-717, 2017.

A. Gershkov, B. Moldovanu, and X. Shi. Voting on multiple issues: What to put on the ballot? Theoretical Economics, 14(2):555-596, 2019.

S. Horan and Y. Sprumont. Two-stage majoritarian choice. 2020.

L. Hurwicz and D. Schmeidler. Construction of outcome functions guaranteeing existence and Pareto optimality of Nash equilibria. Econometrica, 46:1447-1474, 1978.

E. Ianovski and A.Y. Kondratev. Computing the proportional veto core. arXiv preprint arXiv:2003.09153, 2020.

M. Jackson. A crash course in implementation theory. Social Choice and Welfare,, 18:655-708, 2001.

H. Keiding and B. Peleg. Representation of effectivity functions in coalition proof nash equilibrium: A complete characterization. Social Choice and Welfare, 19(2):241-263, 2002.

J.-F. Laslier, M. Núñez, and R. Sanver. A solution to the two-person implementation. Journal of Economic Theory, 194(105261), 2021.

A. Macé and R. Treibich. Inducing cooperation through weighted voting and veto power. American Economic Journal: Microeconomics, forthcoming, 2021.

P. Manzini and M. Mariotti. Sequentially rationalizable choice. American Economic Review, 97(5):1824-1839, 2007.

E. Maskin. Implementation and strong Nash equilibrium. 1978. 
E. Maskin. Nash equilibrium and welfare optimality. Review of Economic Studies, 66:23-38, 1999.

M. Messner and M.K. Polborn. Strong and coalition-proof political equilibria under plurality and runoff rule. International Journal of Game Theory, 35(2):287-314, 2007.

J. Moore and R. Repullo. Nash implementation: A full characterization. Econometrica, 58: 1083-1099, 1990.

H. Moulin. On strategy-proofness and single peakedness. Public Choice, 35:437-455, 1980.

H. Moulin. The proportional veto principle. Review of Economic Studies, 48(3):407-416, 1981.

H. Moulin. Voting with proportional veto power. Econometrica, 50(1):145-162, 1982.

H. Moulin. The Strategy of Social Choice. Advanced Textbooks in Economics. North-Holland, 1983.

D. C. Mueller. Voting by veto. Journal of Public Economics, 10(1):57-75, 1978.

J.F. Nash, R. Nagel, A. Ockenfels, and R. Selten. The agencies method for coalition formation in experimental games. Proceedings of the National Academy of Sciences, 109(50):2035820363, 2012.

M. Núñez and D. Xefteris. Implementation via approval mechanisms. Journal of Economic Theory, 170:169-181, 2017.

D. Ray and R. Vohra. Coalition formation. Handbook of game theory with economic applications, 4:239-326, 2015.

A. Riboni and F. Ruge-Murcia. Monetary policy by committee: consensus, chairman dominance, or simple majority? The Quarterly Journal of Economics, 125(1):363-416, 2010.

A. Riboni and F. Ruge-Murcia. Dissent in monetary policy decisions. Journal of Monetary Economics, 66:137-154, 2014.

T. Saijo, T. Sjostrom, and T. Yamato. Secure implementation. Theoretical Economics, 2:203229, 2007. 
S. Suh. Double implementation in Nash and strong Nash equilibria. Social Choice and Welfare, 14(3):439-447, 1997.

B. Visser and O.H. Swank. On committees of experts. The Quarterly Journal of Economics, 122(1):337-372, 2007.

S. Xiong. Designing referenda: An economist's pessimistic perspective. Journal of Economic Theory, 191:105-133, 2021.

\section{Online Appendix}

This appendix presents an alternative proof of Theorem 1, which shows that a simultaneous veto mechanism coalitionally implements a VRP rule.

\section{C.1 Alternative proof of Theorem 1}

Proposition 3 shows that the set of Nash equilibrium outcomes only includes alternatives from the $\mathrm{VPR}_{v}$ correspondence. It remains to be shown that every alternative in the $\mathrm{VPR}_{v}$ correspondence is a coalition-proof equilibrium outcome of $\mathrm{SV}_{v}$.

To do this we show that, for any $\{a\} \in \operatorname{VPR}_{v}(>)$, it is always possible to construct a strategy profile $m$ which is a coalition-proof equilibrium and selects $a$.

We can formulate the requirement for profile $m$ to be a coalition-proof equilibrium as following (Condition $\eta$ ):

$\eta(i) \bigcup_{i \in I} m_{i}=A \backslash\{a\}$ ( $a$ is the outcome);

$\eta($ ii $) m_{i} \cap m_{j}=\emptyset$ for each $\{i, j\} \in C^{I}$ (no repetitions of vetoes);

$\eta($ iii $)$ For each $J \in C^{I}, U\left(a,>_{I \backslash J}\right) \subseteq m_{J}$ where $U\left(a,>_{I \backslash J}\right)=\bigcap_{j \in I \backslash J} U\left(a,>_{j}\right)$ and $U\left(a,>_{j}\right)=\{b \in A \mid$ $\left.b>_{i} a\right\}$ is the upper contour-set of voter $j$ (any coalition $J$ vetoes the upper contour-set of alternative $a$ for coalition $I \backslash J)$.

The first part is self-explanatory. We have already discussed that at any equilibrium all voters are vetoing different alternatives, thus, $\eta(i i)$ holds. To see why $\eta(i i i)$ is necessary assume that it is violated at some profile $m$. Then there is at least one voter who vetoes an alternative she prefers to $a$ which contradicts Proposition 3. By the same reason any profile 
$m$ satisfying condition $\eta$ is a coalition-proof equilibrium since it rules out self-enforcing deviations.

Consider some alternative $\{a\} \in V P R_{v}(>)$. Note that for any coalition $J$ the following holds:

$$
\left|L\left(a,>_{I \backslash J}\right)\right| \geq \sum_{h \in I \backslash J} v_{h} \Rightarrow\left|U\left(a,>_{I \backslash J}\right)\right| \leq k-\sum_{h \in I \backslash J} v_{h}-1=\sum_{j \in J} v_{j}
$$

Here the left-hand side of (6) follows from the definition of VPR correspondence. The right-hand side of (6) comes from the fact that $\left|L\left(a,>_{I \backslash J}\right)\right|+\left|U\left(a,>_{I \backslash J}\right)\right|=k-1$.

For each $J \in C^{I}$, denote by $Z_{J}=\left(\bigcap_{j \in J} L\left(a,>_{j}\right)\right) \backslash \bigcup_{h \in I \backslash J} L\left(a,>_{h}\right)$ the set of alternatives which are less preferred than $a$ for each voter in $J$ but which are preferred to $a$ for each voter outside $J$. Note that, for each pair $J, K \in C^{I}, Z_{J} \cap Z_{K}=\emptyset$ and $Z_{J} \subseteq U\left(a,>_{I \backslash J}\right)$ for each $J \in C^{I}$.

In what follows we construct a message profile which is a Nash equilibrium of $\mathrm{SV}_{v}$ and leads to the outcome $a$.

For each $i \in I,\left\{m_{i}^{t}\right\}_{t=1}^{t=n-1}$ denotes a sequence defined as follows:

Step 1: for each $i \in I, m_{i}^{1}$ such that $U\left(a,>_{-i}\right) \subseteq m_{i}^{1}$. Note that $m_{i}^{1} \cap m_{j}^{1}=\emptyset$ for each $i, j \in I$ since $a$ is Pareto efficient and hence $U\left(a,>_{-i}\right) \cap U\left(a,>_{-j}\right)=\emptyset$. It follows that the profile $m^{1}$ satisfies condition $\eta(i i)$.

Step 2: for each $i \in I, m_{i}^{2}$ s.t.:

- $m_{i}^{1} \subseteq m_{i}^{2}$ and

- $\forall j \in I: Z_{i j} \subseteq m_{i}^{2} \cup m_{j}^{2}$ with $\left(m_{i}^{2} \cap Z_{i j}\right) \cap\left(m_{j}^{2} \cap Z_{i j}\right)=\emptyset$. This implies that $m_{i}^{2} \cap m_{j}^{2}=\emptyset$ for each $\{i, j\} \in C^{I}$.

Note that profile $m^{2}$ satisfies condition $\eta(i i)$. Also note that $U\left(a,>_{I \backslash\{i, j\}}\right)=U\left(a,>_{-i}\right) \cup U\left(a,>_{-j}\right.$ )$\cup Z_{i j}$. By construction, $U\left(a,>_{-i}\right) \subseteq m_{i}^{1} \subseteq m_{i}^{2}, U\left(a,>_{-j}\right) \subseteq m_{j}^{1} \subseteq m_{j}^{2}$ and $Z_{i j} \subseteq m_{i}^{2} \cup m_{j}^{2}$, thus $U\left(a,>_{I \backslash\{i, j\}}\right) \subseteq m_{i}^{2} \cup m_{j}^{2}$.

For each $t=3, \ldots, n-1$ :

Step $t: m_{i}^{t}$ s.t.:

- $m_{i}^{t-1} \subseteq m_{i}^{t}$ and

- $\forall J \in C^{I}$ s.t. $|J|=t$ and $i \in J, Z_{J} \subseteq \bigcup_{j \in J} m_{j}^{t}$ and $\bigcup_{\{j, h\} \in J, j \neq h}\left(\left(m_{j}^{t} \cap Z_{J}\right) \cup\left(m_{h}^{t} \cap Z_{J}\right)\right)=\emptyset$. 
Note that profile $m^{t}$ satisfies condition $\eta(i i)$. Note that for each $J$ with $|J|=t, U\left(a,>_{I \backslash J}\right)=$ $\bigcup_{K \subseteq J} Z_{K}$. By construction, for all $K \subseteq J, Z_{J} \subseteq \bigcup_{k \in K} m_{k}$. Thus, $U\left(a,>_{I \backslash J}\right) \subseteq \bigcup_{j \in J} m_{j}$.

Note that any profile $m^{n-1}$ satisfies conditions $\eta(i i)$ and $\eta($ iii $)$. We claim that it is always possible to construct a profile $m^{n-1}$ such that $\left|m_{i}^{n-1}\right|=v_{i}$. By contradiction, assume that for any profile $m^{n-1}$ there is at least some voter, say voter $i$, with $\left|m_{i}^{n-1}\right|>v_{i}$. We show that it is possible to construct for each profile $m^{n-1}$, another profile $\widetilde{m}^{n-1}$ such that $\widetilde{m}_{i}^{n-1} \leq v_{i}$ for each $i \in I$ and conditions $\eta(i i)$ and $\eta(i i i)$ are still satisfied.

Observe first that $\sum_{i \in I}\left|m_{i}^{n-1}\right| \leq k-1$. Thus, if $\left|m_{i}^{n-1}\right|>v_{i}$, then there is some voter $j$ with $\left|m_{j}^{n-1}\right|<v_{j}$. There are two possibilities:

- There is some coalition $J$ with $i, j \in J$ such that $m_{i}^{n-1} \cap Z_{J} \neq \emptyset$.

In this case it is possible to reassign some alternatives in $m_{i}^{n-1} \cap Z_{J}$ from voter $i$ to voter $j$ without violating condition $\eta($ iii). In other words we let voter $j$ to veto under profile $\widetilde{m}^{n-1}$ all the alternatives she was vetoing under $m^{n-1}$ plus some alternatives which were vetoed by $i$ under profile $m^{n-1}$. This decreases the number of alternatives assigned to $i\left(\left|m_{i}^{n-1}\right|<\left|\widetilde{m}_{i}^{n-1}\right|\right)$ and increases the number of alternatives assigned to $j$ $\left(\left|\widetilde{m}_{j}^{n-1}\right|<\left|m_{j}^{n-1}\right|\right)$. As was discussed before, condition $\eta(i i i)$ is satisfied independently of which voters from $J$ are vetoing alternatives in $Z_{J}$, thus indeed such reassignment is possible.

- There is no coalition $J$ with $i, j \in J$ such that $m_{i}^{n-1} \cap Z_{J} \neq \emptyset$.

Then we can use intermediate voter $h$ to reassign vetoes. Consider voter $h$ such that:

- there is a coalition $J^{\prime}$ such that $j, h \in J^{\prime}$ and $m_{h}^{n-1} \cap Z_{J^{\prime}} \neq \emptyset$ and

- there is a coalition $J$ such that $i, h \in J$ and $m_{i}^{n-1} \cap Z_{J} \neq \emptyset$.

Then in the same way as in the previous case it is possible to reassign firstly vetoes from voter $h$ to voter $j$, leaving $h$ with less than $v_{h}$ vetoes assigned, and then from voter $i$ to voter $h$.

Note that it is possible that there are more intermediate voters needed. However, the reassignment can be done in the same way and the resulting profile does not violate condition $\eta($ iii), since we reassign alternatives to veto only among voters who have these alternatives in the lower contour-set of $a$ at the same time. 
Thus, combining these cases we can always modify $m$ in such way that conditions $\eta(i i)$ and $\eta\left(\right.$ iii) are satisfied and $\left|\widetilde{m}_{i}^{n-1}\right| \leq v_{i}$ for each $i \in I$.

Finally, note that after satisfying the requirement of conditions $\eta(i i)$ and $\eta($ iii $)$ there still can be some alternatives non-assigned - the alternatives Pareto-dominated by $a$. Indeed, they do not appear in the common upper contour-set of $a$ of any coalition and, thus, are out of the scope of conditions $\eta(i i)$ and $\eta(i i i)$. However, it does not matter which voter is vetoing them, since they are in the lower contour-set of $a$ for all voters. Thus we can assign them to the voters for which $\left|\widetilde{m}_{i}^{n-1}\right|<v_{i}$ after satisfying requirements of conditions $\eta(i i)$ and $\eta(i i i)$. So there is a profile $\widetilde{m}^{n-1}$ which satisfies condition $\eta$ with $\left|\widetilde{m}_{i}^{n-1}\right|=v_{i}$ for each $i \in I$ concluding the proof. 